\title{
Homeostasis of the intervertebral disc requires regulation of STAT3 signaling by the adhesion G-protein coupled receptor ADGRG6
}

Zhaoyang Liu ${ }^{1}$, Garrett W.D. Easson ${ }^{3}$, Jingjing Zhao ${ }^{4}$, Nadja Makki ${ }^{5,4}$, Nadav Ahituv ${ }^{4}$, Matthew

J. Hilton ${ }^{6}$, Simon Y. Tang ${ }^{3}$, and Ryan S. Gray ${ }^{1,2,7, *}$

${ }^{1}$ Department of Nutrional Sciences, University of Texas at Austin, Austin, Texas, USA.

${ }^{2}$ Department of Pediatrics, Dell Pediatric Research Institute, University of Texas at Austin Dell Medical School, Austin, Texas, USA.

${ }^{3}$ Department of Orthopedics, Washington University School of Medicine, Saint Louis, Missouri, USA.

${ }^{4}$ Department of Bioengineering and Therapeutic Sciences and Institute for Human Genetics, University of California San Francisco, San Francisco, California, USA.

${ }^{5}$ Department of Anatomy and Cell Biology, University of Florida, College of Medicine, Gainesville, Florida, USA.

${ }^{6}$ Department of Orthopedic Surgery and Cell Biology, Duke University School of Medicine, Durham, North

Carolina, USA.

${ }^{7}$ Lead Contact

* Correspondence: ryan.gray@austin.utexas.edu

Running Title: ADGRG6 regulates intervertebral disc homeostasis 


\begin{abstract}
Degenerative changes of the intervertebral disc (IVD) are a leading cause of disability affecting humans worldwide. While this is primarily attributed to trauma and aging, genetic variation is associated with disc degeneration in humans. However, the precise mechanisms driving the initiation and progression of disease remain elusive due to a paucity of genetic animal models. Here, we discuss a novel genetic mouse model of endplate-oriented disc degeneration. We show that the adhesion G-protein coupled receptor G6 (ADGRG6) mediates several anabolic and catabolic factors, fibrotic collagen genes, pro-inflammatory pathways, and mechanical properties of the IVD, prior to the onset of overt histopathology of these tissues. Furthermore, we found increased IL-6/STAT3 activation in the IVD and demonstrate that treatment with a chemical inhibitor of STAT3 activation ameliorates disc degeneration in these mutant mice. These findings establish ADGRG6 as a critical regulator of homeostasis of adult disc homeostasis and implicate ADGRG6 and STAT3 as promising therapeutic targets for degenerative joint diseases.
\end{abstract}

Key words: G-protein coupled receptor, disc degeneration, disc herniation, STAT3, scoliosis. 


\section{Author summary}

Degenerative changes of the intervertebral disc (IVD) are a leading cause of disability affecting humans worldwide. While this is primarily attributed to trauma and aging, genetic variation is associated with disc degeneration in humans. However, the precise mechanisms driving the initiation and progression of disease remain elusive due to a paucity of genetic animal models.

Here, we discuss a novel genetic mouse model of endplate-oriented disc degeneration. We show that the adhesion G-protein coupled receptor G6 (ADGRG6) mediates fibrotic collagen expression, causing increased mechanical stiffness of the IVD prior to the onset of histopathology in adult mice. Furthermore, we found increased IL-6/STAT3 activation in the IVD and demonstrate that treatment with a chemical inhibitor of STAT3 activation ameliorates disc degeneration in these mutant mice. Our results demonstrate that ADGRG6 regulation of STAT3 signaling is important for IVD homeostasis, indicating potential therapeutic targets for degenerative joint disorders. 


\section{Introduction}

Spine disorders are one of the most common health issues affecting human populations worldwide, causing a tremendous socio-economic burden. The progression of spine disorders such as low back pain, disc herniation, endplate fracture, and scoliosis are associated with degenerative changes of the intervertebral disc (IVD) (Brinjikji et al., 2015; Dudli et al., 2014; Sun et al., 2017; Zhu et al., 2017b). Therefore, elucidation of the pathways and signaling important for maintaining spine stability and the development and homeostasis of the IVD tissues is critical for the diagnosis, prevention, and treatment of degenerative spine disorders.

The IVD is a fibrocartilaginous joint that connects two adjacent vertebrae and provides structural stability, flexibility, and cushions axial loading of the spinal column (Cortes and Elliott, 2014). The disc is composed of a proteoglycan-rich nucleus pulposus, surrounded by a multi-lamellar annulus fibrosus, and situated between the cartilaginous endplates, which provide nutritional flux to the IVD (Figure 1A). Hallmarks of disc degeneration in humans include loss of disc height, reduced proteoglycan staining, and accumulation of markers of fibrosis within the disc. At the same time, the cartilaginous endplate may show signs of degeneration and calcification, which further compromises nutrient availability to the inner disc layers (Vergroesen et al., 2015). More severe forms of disc degeneration can also result in the herniation of the nucleus pulposus (i) laterally through the annulus fibrosis layer; or (ii) through the cartilaginous endplate into the vertebral body (endplate-oriented), clinically referred to as Schmorl's nodes (Fardon et al., 2014). Genetic susceptibility to disc degeneration has been shown to play a major role in disc degeneration (Sambrook et al., 1999), with the majority of these findings implicating extracellular matrix components of the disc, matrix metalloproteases, or pro-inflammatory cytokines (Munir et al., 2018). Together these data suggest that 
dysregulation of anabolic and catabolic factors as well as inflammatory signaling may underlie many forms of disc degeneration in humans. However, the molecular regulators and initiating factors for these events remain to be defined.

Here, we show that ADGRG6 has a critical role in intervertebral disc homeostasis through regulating STAT3 signaling. ADGRG6 (also called GPR126) is a member of the adhesion G-protein coupled receptor (aGPCR) family of proteins, all of which are thought to have a canonical intercellular signaling function via G-protein coupled signaling, as well as a potential for cell-cell or cell-matrix signaling via the extracellular N-terminal fragment (Patra et al., 2013). In zebrafish, adgrg6/gpr126 is critical for the development of cartilaginous tissues of the semicircular canal via regulation extracellular matrix (ECM) gene expression (Geng et al., 2013), suggesting a role for ADGRG6 in the regulation of cartilaginous tissues. Conditional loss of Adgrg6 in multipotent osteochondral progenitors -giving rise to bone, cartilage, and some connective tissues- of the spine generated postnatal-onset scoliosis, ribcage deformity, and led to midline clefts in the endplates and annulus fibrosus (Karner et al., 2015). Since the development of scoliosis is often linked with IVD deformity (Zhu et al., 2017a), we sought to investigate the role of ADGRG6 in specifically in cartilaginous tissues of the IVD during embryonic and postnatal development.

To define the role of ADGRG6 we combined conditional mouse genetics, genomic approaches, mechanical assessment of intervertebral disc, and cell biological approaches in chondrogenic cell culture. Together, these studies reveal that ADGRG6 has a conserved role for the maintenance of normal chondrogenic gene expression profiles and regulation of STAT3 signaling. We demonstrate that loss of ADGRG6 leads to increased expression of fibrotic collagens and alteration of the normal biomechanical properties of the IVD, prior to the onset of 
endplate-oriented herniations. Finally, we demonstrate loss of ADGRG6 leads to increased, ectopic STAT3 activation in the IVD and that blockade of STAT3 activation can alleviate some degenerative changes of the IVD and progression of endplate-oriented IVD herniation in Adgrg6 conditional mutant mice. Taken together, our work establishes ADGRG6 as a novel regulator of IVD endplate integrity in the mouse and suggests that modulation of ADGRG6/STAT3 signaling could provide robust disease-modifying targets for endplate-oriented disc degeneration, in humans. 


\section{Results}

\section{Loss of ADGRG6 in intervertebral discs leads to endplate-oriented herniations in adult mice}

We have found that conditional removal of ADGRG6 function in the intervertebral disc (IVD) results in the initiation and progression specifically of cartilaginous endplate defects in mice (Figure 1B). Adgrg6 is robustly expressed in the growth plate, but not in cortical or trabecular bone in vertebrae of adult mice by in situ hybridization (Supplemental Figure 1A). The more sensitive fluorescent in situ hybridization (FISH) detection method also established Adgrg6 expression in cells of the cartilaginous endplate, annulus fibrosus, and nucleus pulposus (Supplemental Figure 1C and Figure 1E). To determine the role of ADGRG6 function specifically in these committed chondrogenic lineages of the spine, we utilize an Aggrecan enhancer-driven, Tetracycline-inducible $\underline{\text { Cre }}(A T C)$ transgenic mouse strain (Dy et al., 2012) $\left(A T C ; \operatorname{Adgrg} 6^{f / f}\right)$. We established two experimental groups to address the temporal requirement of ADGRG6 function by induction during embryonic development (from E0.5-P20, prior to IVD specification) or during perinatal development (from P1-P20, after IVD specification).

For both induction strategies, we consistently observed endplate-oriented disc herniations (Adams et al., 2012) in adult mutant mice (Figure 1 and Supplemental Figure 2). ATC;Adgrg6fff mutant mice induced during perinatal developmental displayed prolapse of the nucleus pulposus material into the vertebra body (Figure 1B, D, D'), as did mutant mice recombined during embryonic development (Supplemental Figure 2B, B') ( $n=3$ mice for perinatal induction group; $n=6$ mice for embryonic induction group). These data, suggest that the role of ADGRG for the initiation and progression of IVD defects can largely be attributed to its function during postnatal development. Interestingly, histological analysis of the IVD of $A T C ; A d g r g 6^{f f f}$ mutant mice after 
either induction strategy did not reveal obvious changes in disc height, alterations of overall sulfated-proteoglycan abundance (Safranin-O staining) (Figure 1D, Supplemental Figure 2), or defects of the annulus fibrosis or nucleus pulposus tissues, suggesting a unique endplate-driven pathology for this model.

We found that traditional two-dimensional histological analysis limited our ability to capture the extent and distribution of these endplate-oriented herniations along the spine. To address this, we exploited contrast-enhanced micro-computed tomography $(\mu \mathrm{CT})$ imaging which allows for a full three-dimensional analysis and segmented visualization of the IVD within the intact mouse spine (Lin and Tang, 2017). Reconstruction and segmentation of the whole IVD in 8-month-old ATC;Adgrg6 $6^{f / f}$ mutant (Figure 1H; Movie 1) and Cre (-) control mice (Figure 1G; Movie 2) (49 discs total; $n=5$ mice/genotype) revealed multiple incidences of endplate-oriented disc herniations (yellow arrowheads, Figure 1H). Quantification of the contrast-enhanced $\mu \mathrm{CT}$ imaging indicated from 0-3 herniations present/IVD in ATC;Adgrgfff mutant mice, while Cre (-) control mice did not demonstrate similar defects (Figure 1I). Spatial analysis shows that these herniations occurred along the entire axis of the mutant spine that was imaged (Thoracic (T)5/6 Lumbar (L)3/4), without obvious hotspots. As above, we did not observe radial fissures or lateral prolapse of the disc in $A T C ; A \operatorname{dgrg} 6^{f / f}$ mutant mice using this imaging approach, which further supports that IVD degeneration in these conditional mutant mice are specifically occurring by endplate-driven mechanisms (Adams et al., 2012).

Our previous work demonstrated a clear role for Adgrg6 in the formation of late-onset scoliosis in mouse (Karner et al., 2015), however the cellular pathogenesis of this process remains unresolved. Interestingly, while $\sim 80 \%$ of Col2Cre; Adgrg $6^{f / f}$ mutant mice demonstrated late-onset scoliosis (Karner et al., 2015), we only observed scoliosis in $\sim 12 \%$ of $A T C$;Adgrg6fff 
mutant mice (not shown). One possible explanation is the difference in targeted tissues between the two Cre deleter strains. Analysis of recombination using $\beta$-galactosidase staining of $A T C ;$ Rosa-LacZ mice showed nearly complete recombination throughout the IVD and growth plate at weaning regardless of the timing of induction (Supplemental Figure 3C, D). However, the outer most layers of the annulus fibrosus and the periosteum were not targeted using either strategy (Supplemental Figure 3A, C, D). In contrast, the entire IVD as well as periosteum and trabecular bone in the vertebral body is completely recombined at P1 when crossed to the Col2Cre deleter strain, with marked reduction of recombination in these tissues using the $A T C$ deleter strain (Supplemental Figure 3B, E). However, effective knockdown of Adgrg6 in $A T C ; A d g r g 6^{f / f}$ mutant mice was further confirmed by FISH analysis at 8 months (Figure 1F, Supplemental Figure 1B, D) and by qPCR analysis of gene expression in IVDs extracted from 1.5 month-old mice (Figure 2O). In situ hybridization analysis showed that Adgrg6 expression in non-targeted tissues such as the periosteum was not altered (Supplemental Figure 1E, F). These data suggest that the IVD is important for susceptibility of scoliosis, yet additional effectors of spine stability are involved. However, $A T C$; $A d g r g f / f$ mutant mice consistently exhibited endplateoriented herniations irrespective of whether thoracic scoliosis was observed (Figure 1I and Liu and Gray, unpublished data). These data demonstrate that ADGRG6 has a unique role in the regulation of postnatal homeostasis of the IVD, in addition to scoliosis.

\section{Loss of ADGRG6 in the intervertebral discs leads to alterations of the extracellular matrix, consistent with human disc degeneration pathology}

We next sought to understand the molecular mechanism underlying the initiation of discogenic defects. To guide our analysis, we took cues from molecular changes observed in 
degenerative human IVDs (Yee and Chan, 2014), and revealed several indicators of degenerative joint disease in $A T C ; A d g r g 6^{f / f}$ mutant mice at 1.5 months, prior to overt histopathology (Figure 2D, D', induced from E0.5-P20). Using immunohistochemistry (IHC) we observed reduction in the expression of the transcription factor SOX-9 (SOX9) (Figure 2F, F' and Supplemental Figure 5B) and proteoglycan 4 (PRG4) (Figure 2H and Supplemental Figure 5D) in the inner layer of annulus fibrosus and endplate of the mutant mice. At the same time, expression of the hypertrophic chondrocyte marker type X collagen (COLX) was increased in the endplate and growth plate of the mutant IVD (Figure 2N and Supplemental Figure 5J). We also observed a minor reduction of type II collagen (COLII) staining (Figure 2L and Supplemental Figure 5H) and increased, ectopic expression of matrix metalloprotease-13 (MMP-13) protein in the mutant mice (Figure 2J and Supplemental Figure 5F). Analysis of ATC;Adgrg $6^{\mathrm{f} / f}$ mutant mice induced after birth (P1-P20) demonstrated similar alterations in protein expression in the IVD (Supplemental Figure 4C-J). These results further support the postnatal role for ADGRG6 function in degenerative process of the IVD. Alterations of protein expression were supported by changes in gene expression (Figure 2O), where we observed reduced expression of Col2a1, Sox9 and $\operatorname{Prg} 4$ expression in $A T C ; A d g r g f / f$ mutant mice at 1.5 months. Interestingly, the expression of several catabolic mediators including Mmp13, Adamts4, and Adamts5, as well as markers of dedifferentiation and fibrosis, Collal and Col3al, were increased in the mutant mice. Together, these alterations of typical gene expression profiles in the IVD are consistent with expression profiles reported for degenerative joint diseases, including degenerative discs (Kadow et al., 2015) and osteoarthritis of articulating joints (Kalb et al., 2012; Tonge et al., 2014) in humans.

As discussed above, ADGRG6 is seemingly not critical for early pattering and morphology of cartilaginous tissues of IVD, rather it appears to regulate postnatal and adult 
developmental processes of these tissues. In agreement, we show that embryonic loss of Adgrg6 in cartilaginous tissues does not lead to obvious alternations of the Alcian blue staining or dysmorphology of the disc at P2 (Figure 2B) or at 1.5 months of age (Figure 2D). However, we did observe a mild increase in appearance of acellular clefts in the mutant growth plate $(7.2+3.4$

clefts/IVD; $n=4 ; p=0.03$ ) (Figure 2D-D') at 1.5 months, compared to littermate controls $(2.5 \pm 0.4$ clefts/IVD; $n=4)$ (Figure 2C-C'). This is consistent with our observations of ATC;Adgrg6fff mutant mice, recombined postnatally, which demonstrate minor defects in growth plate at 4months $(40 \% ; n=5)$ (yellow arrows, Supplemental Figure 4B). In addition, TUNEL staining demonstrated a mild increase in cell death in ATC;Adgrg $6^{f / f}$ mutant mice within the annulus fibrosus, nucleus pulposus, and endplate compartments of the IVD, less so within vertebral growth plate at 1.5 months (Supplemental Figure 6B, C). Taken together these data strongly support the critical role of ADGRG6 function in cartilaginous tissues of the IVD during postnatal/adult developmental processes.

\section{Mechanical properties are altered prior to the onset of obvious histopathology in the intervertebral discs of ADGRG6 deficient mice.}

During the progression of disc degeneration and osteoarthritis-related joint remodeling in humans, increased catabolic factors, inflammation, coupled with alterations of normal extracellular matrix composition of the IVD can have deleterious effects on the mechanical properties on these tissues (Nguyen et al., 2017). In order to assess alterations of mechanical properties of $A T C ; A d g r g 6^{f / f}$ mutant IVDs, we isolated individual lumbar discs (Lum.1/2 and 4/5) for dynamic mechanical testing (16 discs total; $n=4$ mice/genotype) from 1.5-month-old mutant and control mice. Using micro-indentation we demonstrated a consistent increase in the stiffness 
(Newton $(\mathrm{N}) / \mathrm{mm}$ ) of $A T C ; A \operatorname{dgrg} 6^{f / f}$ mutant IVDs under 5\% strain cyclic loading (Figure 3A;

$p=0.0114$; mean $\mathrm{w} / 95 \% \mathrm{CI}$ ) and under 50\% monotonic overloading (Figure 3B; $p=0.0026$; mean w/95\% CI). Stiffening of the IVD is commonly observed with early-onset degenerative changes, which compromises the damage resistance of the tissue (Liu et al., 2015a). We analyzed proteoglycan quantification in these IVDs by dimethylmethylene blue assay and found no significant alterations comparing mutant and littermate control mice at 1.5 months. However, we did observed a mild increase in total collagen content in ATC;Adgrg $6^{f / f}$ mutant IVDs by hydroxyproline assay (measured as collagen/wet weight; $p=0.0561$; one-tailed $t$-test), which is consistent with the elevated levels of multiple fibrotic collagen expression in 1.5-month-old mutant IVDs (Figure 2O). We speculate that, alterations in normal extracellular matrix/collagen gene and protein expression, coupled with increased cell death compromise a constellation of factors leading to the decline in the normal mechanical properties of the IVD in ATC;Adgrg6fff mutant mice.

\section{Loss of ADGRG6 in the intervertebral disc leads to increases in fibrotic extracellular matrix genes and alterations in ion transport components.}

To obtain additional, unbiased insights of the molecular and cellular changes in Adgrg6 deficient mice prior to overt disc degeneration, we applied transcriptomic analysis on IVDs of P20 Adgrg6-defeicient mice. To avoid the contamination of untargeted IVD tissue in the $A T C ; A d g r g 6^{f / f}$ mutant mice (e.g. the outer most annulus fibrosus, Supplemental Figure 3C, D), we choose to use Col2Cre; $\operatorname{Adgrg} 6^{f f f}$ mutant mice which completely recombines throughout the entire IVD for these studies (Supplemental Figure 3B, E). Importantly, we observe analogous disc herniations in 8-month-old Col2Cre; Adgrg6 $6^{f / f}$ mutant mice (Supplemental Figure 7). We 
generated three independent libraries for each genotype (Col2Cre;Adgrg $6^{f / f}$ and Cre (-) control) from extracted IVD tissues (T8/9-L4/5), pooled from 2-3 individual mice at P20. We found 884 differential expressed genes with statistical significance ( $p$ value $<0.05$ ) (Supplementary Table 1), and with a more stringent cut-off adjusted $p$ value $<0.05$ and fold-change $\geq 2$, we observed 42 differential expressed genes (Figure 4A). Enriched pathways and biological processes using gene ontology (GO) terms (Huang da et al., 2009) included extracellular matrix, positive regulation of fibroblast proliferation, extracellular matrix structural constituent conferring tensile strength, regulation of tyrosine phosphorylation of STAT protein, and ion transport (Figure 4B and Supplementary Table 2). Several of the significantly upregulated genes are associated with biomarkers or risk of lumbar disc degeneration and osteoarthritis in humans or animal models, including Aspn, Dkk-3, and Mmp3 (Balakrishnan et al., 2014; Kizawa et al., 2005; Mahr et al., 2006; Snelling et al., 2016; Zhang et al., 2016) (Supplementary Table 1). We also observed alterations of chondrogenic and catabolic gene expression (Figure 4C, D). However, surprisingly some of the genes altered in $A T C ; A d g r g 6^{f / f}$ mutant mice at 1.5 months by qPCR analysis (Figure 2O), such as Sox9, Col2a1 and Mmp13, were not similarly changed in slightly younger mice Col2Cre;Adgrg6 $6^{f / f}$ mutant mice (P20) by RNA-Seq analysis. However, in both conditional mutant mice we did observe a consistent upregulation of several fibrillar type collagens, including Colla1, Col3al among others at P20 in Col2Cre; Adgrg6f/f mutant mice (Figure 4E) and Collal and Col3alin ATC;Adgrg $6^{f / f}$ mutant mice at 1.5 months (Figure 2O). Similar shifts from non-fibrous, predominantly type II collagens to fibrillar collagens, such as type I, are common signals reported in studies of degenerative IVD from both human and mouse models (Antoniou et al., 1996; Chen et al., 2016; Yee et al., 2016; Zhang et al., 2018). We suggest that the increased expression of more fibrotic extracellular matrix genes may underlie increased 
stiffness of the IVD we observed in $A T C ; A \operatorname{dgrg} 6^{f / f}$ mutant mice (Figure 3) and contribute to susceptibility of endplate-oriented disc herniations during aging in these mutant mice (Figure 1).

In addition, we observed gene expression changes in several genes associated with ion transport in the IVD of Col2Cre; $A d g r g 6^{f / f}$ mutant mice at P20, including reduced expression of Fxyd2, Capn3, Panx3, and Atp13a4, as well as increased expression of Serpine1, Chrna4, and Slc4al (Figure 4F). High osmotic pressure is a characteristic of the IVD (Fearing et al., 2018) and ion channel activity plays a critical role in the regulation of osmotic changes (Erickson et al., 2001; Matta et al., 2015). In agreement, recent analysis of the SM/J isotype mouse model of disc degeneration mice is associated with gene expression changes in ion transport systems (Zhang et al., 2018). Altogether, our transcriptomic analysis of the IVDs in Col2Cre;Adgrg6 $6^{f / f}$ mutant mice demonstrated a robust dysregulation of several important pathways and components of the IVD homeostasis, including induction of fibrotic gene expression, alteration of ion transport components, as well as changes in some chondrogenic and catabolic factors, prior to the onset of histopathology and disc degeneration. These data strongly suggest that ADGRG6 signaling is a critical regulator of postnatal homeostatic processes of the IVD.

\section{ADGRG6 regulates STAT3 signaling in multiple cartilaginous lineages.}

Our RNA-Seq analyses also implicate pro-inflammatory signaling involved in ADGRG6deficient IVDs. For example, the suppressor of cytokine signaling (Socs) genes, Socs1 and Socs3 were significantly increased in Col2Cre; $\operatorname{Adgrg} 6^{f / f}$ mutant mice (Figure 4G) and implicate pathways associated with inflammation and activation/phosphorylation of STAT proteins (Figure 4B). SOCS3 directly regulates STAT1 (signal transducer and activator of transcription 1) and 
STAT3 activation (Carow and Rottenberg, 2014). For these reasons, we wanted to assay STAT1 and STAT3 activity in the IVD of $A T C ; A d g r g 6^{f / f}$ mutant mice.

By IHC analysis, we observed a substantial increase in phosphorylated STAT3 (pSTAT3) signal, the active form of STAT3 protein in the endplate of the $A T C ; A d g r g 6^{f / f}$ mutant IVD at 1.5 months (Figure 5B, B'), prior to overt histopathology of the disc. Quantification analysis revealed that $16.7 \%$ of cells in the endplate of the mutant IVD are pSTAT3 positive $(n=3$ mice; 2-3 IVDs/mouse; $n=526$ cells total), in comparison to $5.2 \%$ of the control IVD ( $n=3$ mice; $2-3$ IVDs/mouse; $n=417$ cells total) (Figure 5 C). Similar analysis using an antibody against pSTAT1 failed to detect any signal in either genotype (Figure 5D-F). Together these data suggest that ADGRG6 regulates STAT3 activation in endplate of the IVD.

After the induction of cytokines, including interleukin-6 (IL-6), IL-1, Tumor necrosis factor alpha (TNF), STAT3 is phosphorylated by receptor-associated Janus kinases and translocated to cell nucleus to regulate many cellular processes, such as cell growth and apoptosis (Garbers et al., 2015). Moreover, STAT3 activation is implicated for the progression of osteoarthritis (Hayashi et al., 2015; Latourte et al., 2017; Liu et al., 2015b). Our data indicated that ectopic, increased STAT3 activation occurs in young mice prior to histopathology of the mutant IVD, demonstrating a potential role of STAT3 signaling in initiation of endplate-oriented disc degeneration.

In order to facilitate more mechanistic studies of ADGRG6 function in the endplate and growth plate, we utilized the ATDC5 mouse cell line which can be induced to form cartilage-like tissue in vitro (Yao and Wang, 2013). We utilized CRISPR-Cas9 to disrupt the Adgrg6 gene. After screening isolated clones, we identified a stable INDEL mutant with a homozygous 17-bp deletion in exon 3 of Adgrg6 predicted to generate a frameshift mutation at amino acid Ser49 
resulting in a truncated ADGRG6 protein (Adgrg6 $6^{\mathrm{p} . S e r 49+3 f s}$ ) (Figure 6A). The complete reduction of Adgrg6 expression in our clonal Adgrg6 mutant cell line (Adgrg6 KO) suggested a null allele, likely due to non-sense mediated decay of the transcript (Figure 6B). During the course of chondrogenic maturation in unedited ATDC5 cells, Adgrg6 expression increases with similar kinetics as other chondrogenic markers Col2al, Sox9 and Acan (Supplemental Figure 8). Consistent with our observations in vivo (Figure $2 \mathrm{C}-\mathrm{N}$ and $\mathrm{O}$ ), we found decreased expression of several chondrogenic markers, including Col2al and Sox9, and increased expression of hypertrophic marker (CollOal) and catabolic enzyme (Mmp13) in these Adgrg6 KO cells (Figure 6B). In addition, cleaved-Caspase 3, a key effector of apoptosis, was increased 2-fold in Adgrg6 KO cells after 10-day maturation (Supplemental Figure 9), consistent with our observations of increased cell death in $A T C$; $A d g r g 6^{f / f}$ mutant mice IVDs (Supplemental Figure 6). Consistent with the RNA-seq analysis of Col2Cre;Adgrg6f/f conditional mutant mice at P20, we also observed a 5-fold increase in the expression of Socs3 in Adgrg6 KO cells (Figure 6C), while Socs 1 was not detectable in either unedited wild-type or Adgrg6 KO ATDC5 cells (data not shown). Taken together, the strong correlation between these in vivo and in vitro findings suggests a cell autonomous function for ADGRG6 in chondrogenic lineages for the regulation of typical gene expression profiles and of pro-inflammatory signals.

To better understand the intrinsic role of ADGRG6 in regulating STAT3 activation and the inflammatory signaling, we assayed a panel of known pro-inflammatory cytokines $I l 1 a$, Illb, Il6 and Tnf in Adgrg6 KO cells (maturated for 15 days). We observed Il6 expression was the most increased in Adgrg6 KO cells (Figure 6D). Importantly, increased expression of Il6 in the absence of ADGRG6 function was also observed in ATC;Adgrg6fff mutant IVDs at 1.5 months of age (Figure 6E). IL-6 is the major upstream activators of STAT3 signaling (Garbers et al., 2015) 
that has been shown to associate with multiple degenerative joint diseases, including disc degeneration (Wuertz and Haglund, 2013), disc herniation (Eskola et al., 2012), as well as osteoarthritis (Livshits et al., 2009) in humans. IL-6 can not only stimulate the production of catabolic enzymes, but also can suppress the expression of anabolic genes, including Sox9, Col2al, and Acan (Kapoor et al., 2011). To determine whether increased Il6 expression was coupled with activation of STAT3 upon loss of ADGRG6 function, we assayed the ability of ATDC5 cells to respond to recombinant IL-6 protein (rIL-6) in culture. Western blot analysis demonstrated that rIL-6 effectively stimulates the expression of pSTAT3 in both unedited wild type and Adgrg6 KO cells (Figure 6F, G). Interestingly, we detected constitutive pSTAT3 expression in Adgrg6 KO cell lysates, which was further stimulated in expression by the addition of rIL-6 protein (Figure 6F, G). These in vitro results demonstrate that ADGRG6 regulates STAT3 expression and that IL-6 is a potential upstream activator of this signaling pathway in chondrogenic cell culture.

\section{STAT3 blockade protects against disc degeneration and herniation.}

To define the role of STAT3 signaling on the pathogenesis of endplate-oriented disc degeneration, we next tested if inhibition of STAT3 activation in the Col2Cre;Adgrg6fff mutant mouse model which displays disc herniations by 8 -months-of-age. To test this we utilized the constitutive Col2Cre; $A d g r g 6^{f / f}$ conditional mutant in order to limit stress on the mice by avoiding exposure to Dox, as potential confounding variables, prior to long-term treatment with the STAT3 inhibitor, Stattic. Importantly, Col2Cre;Adgrg $6^{f / f}$ mutant mice exhibit similar phenotypes as observed in $A T C ; A \operatorname{dgrg} 6^{f / f}$ mutant mice, including: erosion and clefts in the endplate and growth plate (yellow arrowheads; Figure 7B' and supplemental Figure 8), ectopic expression of 
COLX in the endplate (red arrows; Figure 7E), increases in the appearance of endplate-oriented herniations with average of 9.6 herniations/mouse ( $n=3$ mice; 39 IVDs) (Figure 7G). We treat both control and mutant groups with either a small molecule named Stattic, a nonpeptidic STAT3 inhibitor (25mg/kg, dissolved in DMSO), (Schust et al., 2006), or placebo (DMSO/PBS/Tween-20) via i.p. injection for 16 weeks beginning by the age of 1.5 months. Mutant mice receiving Stattic treatment displayed a reduction in endplate defects with an average of 3 herniations /mouse ( $n=3$ mice; 39 IVDs) (Figure 7C, C' and G). One way ANOVA followed by Tukey HSD test indicated that the mean score for the Stattic-treatment condition $((\mathrm{M})$ ean $=0.23, \mathrm{SD}=0.54)$ was significantly different $(p<0.01)$ than the placebo-treated Col2Cre;Adgrg $6^{f f f}$ mutant mice $(\mathrm{M}=0.74, \mathrm{SD}=0.56)$, but did not differ significantly from the placebo-treated Cre $(-)$ control littermates $(\mathrm{M}=0.096, \mathrm{SD}=0.09)$. Notably, placebo-treated Cre (-) control mice also display minor endplate defects with an average of 1.25 herniations/ mouse ( $n=4$ mice; 52 IVDs), which is not observed in Cre (-) controls in Figure 1I. This may due to (i) DMSO content in the placebo treatment and (ii) different genetic background between ATC;Adgrg $6^{f / f}$ and Col2Cre;Adgrg $6^{f / f}$ mice. Of note, Col2Cre;Adgrg $6^{f / f}$ mutant mice receiving Stattic treatment displayed normal COLX expression in the endplate compared with the placebotreated mutant mice (Figure 7E, F). In contrast, Stattic treatment had little effect on the general reduction of COLII and SOX9 expression in Col2Cre;Adgrg $6^{f / f}$ mutant mice (Supplemental Figure 10). These data suggest that additional effectors of ADGRG6 function, apart from STAT3 signaling, are required for maintenance of normal gene expression profiles in cartilaginous tissue of the IVD. In conclusion, these results suggest that blockade of STAT3 singling has a protective effect for endplate-oriented herniation, potentially through the repression of ectopic, increased COLX expression in the absence of ADGRG6 function. 
Taken together, our studies demonstrate that ADGRG6 has a cell autonomous role in the regulation of STAT3 signaling in chondrogenic lineages and with the IVD. Our demonstration that the small molecule STAT3 inhibitor Stattic can alleviate the onset and progression of degenerative changes and the formation of the endplate-oriented herniations in the IVDs of Adgrg6-deficient mice, implicating dysregulation of STAT3 signaling may underlie some forms of disc degeneration in particular for endplate-oriented disc degeneration. This implicates ADGRG6/STAT3 signaling pathway as a potential target for therapeutic approaches to treat disc degeneration, and possibly other joint degeneration disease, including osteoarthritis. 


\section{Discussion}

In this study, we demonstrate for the first time that ADGRG6 signaling acts as a positive regulator of postnatal homeostatic mechanisms of the IVD. This function is demonstrated by global changes in gene expression profiles in the IVD including alterations of several anabolic and catabolic factors and ion transport components, increased expression of fibrillar collagens and pro-inflammatory cytokine signaling, which occur several months prior to obvious histopathology of the disc in adult mice. We suggest that these alterations also lead to a general stiffening of the intervertebral disc, which synergistically contribute to the formation of endplate defects of the disc over the course of adult development.

In addition, we demonstrate that ADGRG6 acts as a cell autonomous regulator of the cytokine-induced STAT3 signaling in the cartilaginous endplate of the IVD, and that inhibition of this signaling provides protective effects against the onset and severity of endplate-oriented disc herniations due to the loss of ADGRG6 function. It will be interesting to test if blockade of STAT3 signaling has a general protective effect of endplate integrity that is not dependent on ADGRG6 function. However, to our knowledge there is not a well-established alternative experimental mouse model of endplate-oriented disc herniations with which to test this. It will be important to determine how STAT3 signaling may drive other models of disc degeneration characterized by lateral herniations and/or pathology of the nucleus pulposus.

The role of ADGRG6 appears to be dispensable for most developmental processes of cartilaginous tissues of the spine (Figure 2B). In contrast, we revealed a novel role for ADGRG6 function in the postnatal regulation of gene expression, stiffening of the IVD, and endplate-plate oriented disc defects in adult mice. Interestingly, we demonstrated that ADGRG6 is important for maintaining normal expression of SOX9, a master transcription factor for both 
chondrogenesis during embryonic development (Akiyama et al., 2002) and cartilage maintenance during postnatal development (Henry et al., 2012), in the IVD. This regulation of SOX/Sox 9 by of ADGRG6 function was recapitulated in heterologous chondrogenic ATDC5 cell culture, where we observed reduced expression of $\operatorname{Sox} 9$ along with its direct target gene Col2al. Conservation of this function is also observed in the cartilaginous semicircular canal of adgrg6/gpr126 mutant zebrafish which similarly display altered expression of several extracellular matrix genes as well as decreased expression of sox $9 b$ (Geng et al., 2013). Furthermore, genetic ablation of $\operatorname{Sox} 9$ in adult mice demonstrates reduction of Adgrg6/Gpr 126 expression, as well as many similarities as reported in this study including increased cell death and alterations of extracellular matrix gene expression (Henry et al., 2012). However, in contrast to our observations in Adgrg6-deficient mice, these Sox9-deficient mice also exhibit decreased disc height and loss of proteoglycan in the IVD (Henry et al., 2012). This may be explained by a more gradual loss of SOX9 expression in our conditional Adgrg6 mouse model, which may stimulate unknown compensatory mechanisms that may overcome the onset of proteoglycan depletion observed in Sox9-deficent mice (El-Brolosy and Stainier, 2017). In addition, the ablation of Adgrg6 in the IVD leads to unique endplate defects and alterations of a distinct suite of gene expression profiles, which was not reported after genetic ablation of Sox 9 in adult mice (Henry et al., 2012). Despite these observations, our study suggests a degree co-regulation exists for the expression of Adgrgr6 and Sox9. The identification of the factors that govern this regulation and how this mechanistically contributes during homeostasis and disease warrants further investigation.

Our findings support a model where increased expression fibrillar collagens in IVD tissues in Adgrg6 deficient mice precede the onset of increased disc stiffness and failure of the 
endplate. Using contrast-enhanced $\mu \mathrm{CT}$ imaging of the intact mouse spine, we for the first time describe the appearance and distribution of the endplate-oriented herniations in Adgrg6 conditional mutant mice. Specifically, this mouse model of disc degeneration displays an upregulation of multiple fibrillar collagen genes (e.g. Colla1, Col3a1, Col4a1, and Col5a1) in the IVDs at a juvenile stage of development (P20). Increased expression of fibrillar collagens and induced stiffness of the IVD is expected to result in disturbed stress distribution of the IVD, concentrate loading at the cartilaginous endplate, which increase the risk of endplate fractures and the formation of disc herniations (Adams et al., 1996; Vergroesen et al., 2015; Wilke et al., 2016). The adult IVD is thought to be an avascular tissue, as such its major source of nutrient flux occurs via diffusion through endplate (Urban et al., 2004). Increased expression of fibrillar collagens and catabolic enzymes in the disc including ectopic over-expression of COLX in the endplate are correlated with endplate sclerosis and reduced nutrient supply (Smith et al., 2011; Zhao et al., 2007). In this way a viscous cycle comprising increased expression of catabolic enzyme activity, pro-inflammatory factors, and apoptosis, can further exacerbate the integrity of the IVD leading to endplate-oriented herniation (Vergroesen et al., 2015). In conclusion, we suggest that increased fibrillary collagen expression and stiffness of the disc, increased ectopic expression of COLX in the endplate coupled with increases other catabolic factors such as MMPs and ADAMTSs, synergistically contribute to the endplate-specific defects in this mouse model.

Our previous work demonstrated a clear role for Adgrg6 in the formation of scoliosis in mouse (onset at around P20-P40) (Karner et al., 2015). Here we show additional defects of endplate-oriented disc herniations in both Col2Cre- and ATC;Adgrg $6^{f / f}$ mutant mouse models (onset at around 6 to 8 months of age), which are reminiscent of an idiopathic human condition 
called acute Schmorl's nodes (Adams and Dolan, 2012). While increased co-occurrence of Schmorl's nodes in idiopathic scoliosis patients has been described (Buttermann and Mullin, 2008), additional studies in a larger cohort of AIS patients are needed to understand if an association between these IVD pathologies is relevant in the pathogenesis of disease in humans. Interestingly, while the incidence of scoliosis in Col2Cre- and ATC;Adgrg $6^{f / f}$ mutant mice was $\sim 80 \%$ and $\sim 12 \%$ respectively, herniations were observed in $\sim 100 \%$ of mutant mice in both models (Liu and Gray, unpublished data, Figure 1I and Figure 7G). Scoliosis in these mutant mice is specifically associated with curvature in the thoracic spine, while endplate-oriented herniations were observed along the entire spine axis. Taken together, our observations suggest that the mechanisms which promote the formation of endplate-oriented disc herniations in adult mice are partially independent from the pathogenesis of postnatal-onset idiopathic scoliosis in these mutant mice. It is tempting to speculate that alterations of the mechanical properties of the IVD during postnatal development underlie the susceptibility of scoliosis at this stage of development. However, additional and ongoing studies are required to better understand the cellular pathogenesis underlying scoliosis in Adgrg6 mutant mice.

We demonstrate an upregulation of IL-6/STAT3 signaling, coupled with increased Socs3 expression in Adgrg6 conditional mutant mice, prior to overt histopathology. Furthermore, analysis of Adgrg6-KO ATDC5 cells in culture show activation of the IL-6/STAT3/Soc3 pathway is an intrinsic property of cartilaginous cells, regulated by ADGRG6 function. Interestingly, IL-6/STAT3 signaling has been demonstrated to promote chondrogenic differentiation of human mesenchymal stem cells (Kondo et al., 2015), on the other hand IL6/STAT3 signaling has been implicated in both degenerative disc disease and osteoarthritis. For example, induced expression of IL-6 and activation of STAT3 (pSTAT3) has been observed in 
human patients with disc degeneration and disc herniation (Osuka et al., 2014; Suzuki et al., 2017). Moreover, circulating IL-6 is positively associated with radiographic osteoarthritis and cartilage loss of the knee in humans (Stannus et al., 2010), and IL-6/STAT3 signaling is activated in trauma-induced osteoarthritis (Latourte et al., 2017; Liu et al., 2015b). IL-6 can also stimulate the expression of catabolic markers, such as COLX and MMP13 which are commonly associated with degenerative joint disease (He et al., 2014; Hunter et al., 2014). Importantly, systemic inhibition of STAT3 signaling with Stattic (Schust et al., 2006) can alleviate the onset and progression of joint remodeling in a post-traumatic osteoarthritis model in mouse (Latourte et al., 2017). However, how STAT3 activation is related to the initiation and progressions of these joint degenerative disorders remains to be determined. Nevertheless, we were able to demonstrate the alleviation of some aspects of endplate-oriented disc degeneration caused by Adgrg6-deficiency in the IVD with STAT3 inhibitor (Stattic) treatment. Altogether, this study provides clear evidence of the therapeutic value of STAT3 signaling for the onset and progression of disc degeneration.

Our work of comprehensive analysis of IVD development with both embryonic and postnatal deletion of Adgrg6 also provide the first glimpse into the function and signal properties of an adhesion G-Protein coupled receptor (aGPCR) in this process. aGPCRs can function as classical GPCRs to invoke G-protein dependent intracellular cis signaling (Hamann et al., 2015). Indeed, ADRGR6 signals through Gs-protein/cAMP (Liebscher et al., 2014; Mogha et al., 2013). Future efforts will seek to analysis of how stimulation of Gs-signaling may alleviate IVD pathology and cAMP responsive binding elements are associated with alterations of genes affected in these Adgrg6 deficient models. Given that GPCRs are among the most druggable classes of proteins, our work presented here identify a potential therapeutic target for 
bioRxiv preprint doi: https://doi org/10.1101/581595; this version posted March 18, 2019. The copyright holder for this preprint (which was not certified by peer review) is the author/funder, who has granted bioRxiv a license to display the preprint in perpetuity. It is made available under aCC-BY 4.0 International license.

degenerative joint diseases, and should encourage the targeted survey of the ADGRG6 locus in human cohorts of disc degeneration and osteoarthritis. 


\section{Methods}

\section{Mouse Strains}

All animal studies and procedures were approved by the Animal Studies Committee at the University of Texas at Austin. Mice were housed in standard cages and maintained on a 12-hour light/dark cycle, with rodent chow and water available ad libitum. All mouse strains were described previously, including Adgrg6fff (Taconic \#TF0269) (Mogha et al., 2013); Rosa26;LacZ (B6;129S-Gt(ROSA)26Sor/J) (Soriano, 1999); ATC (Dy et al., 2012), and Col2Cre (Long et al., 2001). Doxycycline (Dox) was administered to $A T C$; $A \operatorname{dgrg} 6^{f / f}$ mice and littermate controls with two strategies: (i) inducing from embryonic day (E)0.5-postnatal day (P)20 by ad libitum feeding of Dox-chow (Test Diet, 1814469) to plugged isolated females, and supplemented with intraperitoneal (IP) injections of the pregnant dames once/week (10mg Dox/kg body weight) throughout the pregnancy until the pups were weaned at P20; (ii) inducting from P1-P20 by ad libitum feeding of Dox-chow to the mothers after the pups were born, and supplemented with intraperitoneal (IP) injections of the mothers once/week (10mg Dox/kg body weight) until the pups were weaned at P20. ATC; Rosa-LacZ ${ }^{f /+}$ mice were induced with the same strategies.

STAT3 inhibitor Stattic (25mg/kg, dissolved in DMSO) or placebo (DMSO/PBS/Tween-20) were administered to Col2Cre; Adgrg6 f/f mutant mice or Cre (-) littermate controls via i.p. injection once/week for 16 weeks beginning by the age of 1.5 months. Mice were harvested at P2, P20, 1.5 months, 6 months and 8 months of age.

\section{Analyses of mice}

Histological analysis was performed on thoracic spines fixed in 10\% neutral-buffered formalin for 3 days at room temperature followed by 1-week decalcification in Formic Acid Bone 
Decalcifier (Immunocal, StatLab). After decalcification, bones were embedded in paraffin and sectioned at $5 \mu \mathrm{m}$ thickness. Safranin O/Fast Green (SO/FG) and Alcian blue Hematoxylin/Orange G (ABH/OG) staining were performed following standard protocols (Center for Musculoskeletal Research, University of Rochester). Immunohistochemical analyses were performed on paraffin sections with traditional antigen retrieval and colorimetric development methodologies with the following primary antibodies: anti-Collagen II (Thermo Scientific, MS235B), anti-Collagen X (Quartett, 1-CO097-05), anti-SOX9 (Santa Cruz Biotechnology, sc-20095), anti-Lubricin (PRG4) (Abcam, ab28484), anti-MMP-13 (Thermo Scientific, MS-825-P), anti-IL-6 (Abcam, ab6672), and anti-phospho-STAT3 (Cell Signaling, \#9145). The Terminal deoxynucleotidyl transferase dUTP Nick-End Labeling (TUNEL) cell death assay was performed on paraffin sections with the In Situ Cell Death Detection Kit, Fluorescein (Roche) according to the manufacturer's instructions. The b-galactosidase staining was performed on frozen sections as previously described (Liu et al., 2015b). Spines were harvested and fixed in $4 \%$ paraformaldehyde for 2 hours at $4{ }^{\circ} \mathrm{C}$ and decalcified with $14 \%$ EDTA at $4{ }^{\circ} \mathrm{C}$ for 1 week. Tissues were washed in sucrose gradient, embedded with Tissue-Tek OCT medium, snap-frozen in liquid nitrogen, and sectioned at $10 \mu \mathrm{m}$ with a Thermo Scientific HM 550 cryostat. In situ hybridization using a Digoxygenin-labeled antisense riboprobe for Adgrg6 was performed on $5 \mu \mathrm{m}$ paraffin sections as described previously with modifications (Karner et al., 2015), and detected with either a chromogenic substrate (BM Purple, Roche) or a tyramineamplified fluorescent antibody (Perkin Elmer).

\section{Cell culture}


ATDC5 cells (Sigma, 99072806) were maintained in DMEM/F-12 (1:1) medium (Gibco, 11330032) supplemented with 5\% FBS and 1\% penicillin/streptomycin. ATDC5 cells were differentiated in DMEM/F-12 (1:1) medium supplemented with 5\% FBS, $1 \%$ penicillin/streptomycin, 1\% ITS premix (Corning, 354352), 50 $\mathrm{gg} / \mathrm{ml}$ ascorbic acid, $10 \mathrm{nM}$ dexamethasone, and 10ng/ml TGF- 33 (Sigma, SRP6552) for 5, 10, and 15 days.

Both wild type and Adgrg6 KO ATDC5 cells were treated with 100ng/ml recombinant human IL-6 protein (rIL-6) (R\&D System, 206-IL) for 2 hours before protein extraction.

\section{Generation of the Adgrg6 KO cell line}

CRISPR reagents were generated to target the 3rd exon of mouse Adgrg6

(ENSMUST00000041168.5) using the following oligos: Adgrg6-g33-fwd ACACCGAGGGTAACACGGAGACGTAAG and Adgrg6-g33-rev AAAACTTACGTCTCCGTGTTACCCTCG and cloned into a lentiviral packing vector (lentiCRISPR v2 was a gift from Feng Zhang (Addgene plasmid \# 52961)) along a pCas9_GFP (a gift from Kiran Musunuru (Addgene plasmid \# 44719)). Lentiviral particle packaging was in A293T cells using standard 3rd generation approach (https://tronolab.epfl.ch/page-148635en.html). Human embryonic kidney (HEK) 293T cells (Sigma) were maintained in DMEM supplemented with 10\% fetal bovine serum, 2mM GlutaMAX (Life Technologies), 100U/ml penicillin, and $100 \mathrm{ug} / \mathrm{mL}$ streptomycin at $37^{\circ} \mathrm{C}$ with $5 \% \mathrm{CO}_{2}$ incubation. 293T cells were seeded into $6 \mathrm{~cm}$ plates (Corning) one day prior to transfection at a density of $2 \times 10^{6}$ cells per well. 293T cells were transfected using FUGENE 6 (Promega) following the manufacturer's recommended protocol. For each plate, a total of $0.5 \mathrm{ug}$ of each plasmid was used. At 2 and 4 days post 
transfection, the cell media was collected and filtered with $0.45 \mu \mathrm{M}$ filter (Corning) and stored at $-80^{\circ} \mathrm{C}$

ATDC5 were plated in 6-well plates to $80 \%$ confluency and lentiviral transduction was using diluted viral media with $0.1 \%$ polybrene (EMD Millipore) for 24 hours followed by selection with $4 \mu \mathrm{g} / \mathrm{ml}$ Blastocidin and Puromycin for 5 days post transfection. Serial dilution under selection was used to identify individual clones, expanded colonies were screened for INDEL mutations using Adgrg6-ex3-fwd - TTGACAGTTACTGCTTGATGCCCCC and Adgrg6-ex3-rev- CCCTTGGCAGTCGCTCCACAGAATT primers and amplicons were screen by Sanger sequencing to identify homozygous clones.

\section{RNA isolation and qPCR}

Intervertebral discs from the thoracic and lumbar spine (T8-L5) of the 1.5-month old ATC; Adgrg6fff and control mice were isolated in cold PBS, snap frozen and pulverized in liquid nitrogen. Total RNA from intervertebral discs was isolated using the TRIzol Reagent (Invitrogen, 15596026), and cleaned up with the Direct-zol RNA miniprep kit (Zymo Research, Z2070). Total RNA of the cultured ATDC5 cells was isolated using the RNAeasy mini kit (Qiagen, 74104). Reverse transcription was performed using $1 \mu \mathrm{g}$ total RNA with the iScript cDNA synthesis kit (BioRad). Reactions were set up in technical and biological triplicates in a 96 well format on an BioRAD CFX96 real-time PCR detection system, using SYBR green chemistry (SsoAdvanced, BioRad). The PCR conditions were $95^{\circ} \mathrm{C}$ for 3 min followed by 40 cycles of $95^{\circ} \mathrm{C}$ for $10 \mathrm{~s}$ and $58^{\circ} \mathrm{C}$ for $30 \mathrm{~s}$. Gene expression was normalized to $b$-actin mRNA and relative expression was calculated using the $2^{-(\Delta \Delta C t)}$ method. All qPCR primers sequences are 
listed in Supplementary Table 3. PCR efficiency was optimized and melting curve analyses of products were performed to ensure reaction specificity.

\section{RNA isolation, library construction and sequencing}

Intervertebral discs from the thoracic and lumbar spine (T8-L5) of the P20 Col2Cre; Adgrg6fff and control mice were isolated in cold PBS, snap frozen and pulverized in liquid nitrogen. Total RNA was extracted using Trizol reagent (Invitrogen, CA, USA) following the manufacturer's procedure. The total RNA quantity and purity were analyzed on Bioanalyzer 2100 and RNA 6000 Nano LabChip Kit (Agilent, CA, USA) with RIN number >7.0. Total RNA was subjected to isolate Poly (A) mRNA with poly-T oligo attached magnetic beads (Invitrogen). RNA fragments were reverse-transcribed to create the final cDNA libraries following the NEBNext ${ }^{\circledR}$ Ultra $^{\mathrm{TM}}$ RNA Library Prep Kit (Illumina, San Diego, USA), paired-end sequencing was performed.

\section{Bioinformatics analysis}

\section{Transcripts Assembly:}

Firstly, Cutadapt (Martin, 2011) and perl scripts in house were used to remove the reads that contained adaptor contamination, low quality bases and undetermined bases. Then sequence quality was verified using FastQC (http://www.bioinformatics.babraham.ac.uk/projects/fastqc/). We used HISAT2 (Kim et al., 2015) to map reads to the genome of Mus Musculus (GRCm38.88). The mapped reads of each sample were assembled using StringTie (Frazee et al., 2015). Then, all transcriptomes from biological samples were merged to reconstruct a comprehensive transcriptome using perl scripts and gffcompare. After the final transcriptome 
was generated, StringTie (Pertea et al., 2015) and Ballgown (Frazee et al., 2015) was used to estimate the expression levels of all transcripts.

\section{Different expression analysis of mRNAs:}

StringTie (Pertea et al., 2015) was used to perform expression level for mRNAs by calculating FPKM. The differentially expressed mRNAs were selected with $\log 2$ (fold change) $>1$ or $\log 2$ (fold change) $<-1$ and with statistical significance $(\mathrm{p}$ value $<0.05)$ by $\mathrm{R}$ package Ballgown (Frazee et al., 2015).

\section{Western blotting}

For western blotting analysis, total proteins were extracted from cells with protein extraction buffer [50mM HEPES, 1.5mM EDTA (pH 8.0) , 150mM NaCl, 10\% glycerol, 1\% Triton X-100] supplemented with protease and phosphatase inhibitors (Roche). 10mg of protein from each sample was resolved by $4-15 \%$ SDS-polyacrylamide gel electrophoresis and transferred to the nitrocellulose membranes. Western blots were then blocked with LI-COR blocking buffer and incubated overnight with primary antibodies anti-STAT3 (Cell Signaling, \#4904), anti-pSTAT3 (Cell Signaling, \#9145), and anti-GAPDH (Cell Signaling, \#2118) at $4{ }^{\circ} \mathrm{C}$ with gentle rocking. The next day western blots were detected with the LI-COR Odyssey infrared imaging system.

\section{Contrast-enhanced $\mu \mathrm{CT}$ imaging and segmentation}

Samples undergoing contrast-enhanced micro-computed tomography $(\mu \mathrm{CT})$ were incubated in a 35\%w/v solution Ioversol in PBS (OptiRay 350, Guerbet, St. Louis) supplemented with 1\% penicillin-streptomycin at $37 \mathrm{C}$ one day prior to scanning. Immediately prior to scanning, the sample was removed from the solution and wrapped in PBS-soaked gauze. These samples were 
mounted in $2 \%$ agarose gels and then scanned using the microCT40 system (Scanco Medical, $\mathrm{CH})$ operating at $6 \mu \mathrm{m}$ voxel size $(45 \mathrm{kVp}, 177 \mathrm{uA}, 300 \mathrm{~ms}$ integration). Following our previous method for segmentation of murine IVDs (Lin and Tang, 2017; Lin et al., 2016), the $\mu$ CT CT data is exported as a DICOM file for further processing. Following an initial median filter (sigma $=0.8$, support $=3$ ), bone is then thresholded out, and the soft tissue not part of the IVD was removed by drawing contours around the outer edge of every five transverse slices of the AF and morphed using a linear interpolation. The remaining voxels are designated as the whole disc mask. From the masks of the whole disc, volumes and average attenuations (intensity) are calculated. The volume was determined from the total number of voxels contained within the mask and the attenuation is taken as the average 16-bit grayscale value of the voxels.

Visualizations of the $\mu \mathrm{CT}$ were obtained using OsiriX (Pixmeo, Geneva). The volume of the contoured disc was then measured. Defects of the IVD resembling Schmorl's Nodes were defined by three or more consecutive slices that had a rupture in the same area of the endplate. This method was chosen so as to eliminate any potential spatial artifacts that may be misidentified as ruptures.

\section{Mechanical testing}

The mechanical properties of the isolated intervertebral discs were determined using dynamic compression on a microindentation system (BioDent 1000; Active Life Scientific, Santa Barbara, CA) with a $2.39 \mathrm{~mm}$ non-porous, flat probe (Liu et al., 2015a). The probe's load cell resolution is $0.001 \mathrm{~N}$, and the system's Piezo actuator resolution is $0.01 \mu \mathrm{m}$. Each sample was moved into position under the probe tip by gripping the aluminum platen. The indenter tip was aligned over each sample so that the probe covered the entire diameter of the disc. Each disc was first loaded 
sinusoidally at $10 \%$ strain peak strain at $1 \mathrm{~Hz}$ for 20 cycles with a $0.1 \mathrm{~N}$ preload. After the cyclic tests, the discs were monotonically overloaded to $50 \%$ strain at a loading rate of $10 \%$ strain per second. The loading slope value was obtained from the linear region of the force displacement curve from all loading curves. These samples were maintained in physiological PBS solution (pH 7.2) during and between trials to simulate the osmotic pressures found in the body and maintain hydration of the IVD.

\section{Quantification of collagen and proteoglycans}

The wet weight of each isolated disc was taken after mechanical testing utilizing an analytical balance (A-200DS; Denver Instrument Company, Bohemia, NY). Samples were first digested in papain at $65^{\circ} \mathrm{C}$ for $18 \mathrm{~h}$. The samples were then centrifuged and the supernatant collected and then plated in triplicate. Proteoglycan content was quantified using the colorimetric dimethylmethylene blue (DMMB) assay (Sabiston et al., 1985) by measuring the absorbance 525nm with chondroitin sulfate from bovine cartilage as standards (Sigma-Aldrich, St. Louis, MO), and then normalized to wet weight of the IVD. The remaining papain-digested lysates were then used for hyproxyproline quantification. The amount of collagen was approximated by assuming that hydroxyproline accounts $1 / 7$ of the mass of collagen. The samples were hydrolyzed in $12 \mathrm{~N}$ hydrochloric acid at $120^{\circ} \mathrm{C}$ for $3 \mathrm{~h}$. The hydrolyzed samples were then plated in triplicates. A chloramine T colorimetric assay (Woessner, 1961) and standardized using hydroxyproline by quantifying the absorbance at $560 \mathrm{~nm}$ using a plate reader (SpectraMax M2, Molecular Devices, Sunnyvale, CA). Quantification of proteoglycan content in ATDC5 cell culture were conducted as previously described with modifications (Kitaoka et al., 2001). Briefly, ATDC5 cells were fixed with $10 \%$ neutral buffered formalin for 10 minutes at room temperature and incubated with 
$3 \%$ acetic acid for 10 minute, followed by staining with $1 \%$ Alcian blue (in $3 \%$ acetic acid, $\mathrm{pH}$

2.5) for 30 minutes at room temperature. Cells were washed with PBS and air-dried overnight.

After taking pictures, Alcian blue was extracted with 500ul of DMSO and quantifying the

absorbance at $650 \mathrm{~nm}$ using a plate reader. At least three biological replicates were analyzed for each experimental group.

\section{Statistics}

Statistical analyses to compared the mutant and control groups were performed using 2-tailed

Student's $t$-test and one-way ANOVA followed by Turkey HSD test as appropriate (GraphPad

Prism 7). A $p$ value of less than 0.05 is considered statistically significant. 


\section{Author contributions}

ZL and RSG conceived the experiments. ZL, GE, and NM performed the experiments. ZL, NM, and JZ performed RNA-sequencing and analysis. RSG, SYT, NM, NA and MJH provided reagents, expertise, and feedback. ZL and RSG wrote the manuscript. 


\section{Acknowledgements}

We would like to thank Dr. Fanxin Long and Dr. Veronique Levebre for sharing the Col2Cre and $A T C$ mouse strains respectively. We would like to thank Drs. John Wallingford and Steve Vokes, and members of the Gray lab for helpful comments on this manuscript prior to submission. Research reported in this publication was supported by the National Institute of Arthritis and Musculoskeletal and Skin Diseases of the National Institutes of Health under Award Number R01AR072009-01 (R.S.G.), F32AR073648 (Z.L.), R21AR069804 (S.T.), R01AR063071 (M.J.H) and the National Institute of Child \& Human Development 1P01HD084387 (N.A). 


\section{References}

Adams, M., Simms, R. J., Abdelhamed, Z., Dawe, H. R., Szymanska, K., Logan, C. V., Wheway, G., Pitt, E., Gull, K., Knowles, M. A., et al. (2012). A meckelin-filamin A interaction mediates ciliogenesis. Hum Mol Genet 21, 1272-1286.

Adams, M. A. and Dolan, P. (2012). Intervertebral disc degeneration: evidence for two distinct phenotypes. J Anat 221, 497-506.

Adams, M. A., McMillan, D. W., Green, T. P. and Dolan, P. (1996). Sustained loading generates stress concentrations in lumbar intervertebral discs. Spine (Phila Pa 1976) 21, 434-438.

Akiyama, H., Chaboissier, M. C., Martin, J. F., Schedl, A. and de Crombrugghe, B. (2002). The transcription factor Sox 9 has essential roles in successive steps of the chondrocyte differentiation pathway and is required for expression of Sox5 and Sox6. Genes Dev 16, 2813-2828.

Antoniou, J., Steffen, T., Nelson, F., Winterbottom, N., Hollander, A. P., Poole, R. A., Aebi, M. and Alini, M. (1996). The human lumbar intervertebral disc: evidence for changes in the biosynthesis and denaturation of the extracellular matrix with growth, maturation, ageing, and degeneration. J Clin Invest 98, 996-1003.

Balakrishnan, L., Nirujogi, R. S., Ahmad, S., Bhattacharjee, M., Manda, S. S., Renuse, S., Kelkar, D. S., Subbannayya, Y., Raju, R., Goel, R., et al. (2014). Proteomic analysis of human osteoarthritis synovial fluid. Clin Proteomics 11, 6.

Brinjikji, W., Diehn, F. E., Jarvik, J. G., Carr, C. M., Kallmes, D. F., Murad, M. H. and Luetmer, P. H. (2015). MRI Findings of Disc Degeneration are More Prevalent in Adults with Low Back Pain than in Asymptomatic Controls: A Systematic Review and Meta-Analysis. AJNR Am J Neuroradiol 36, 2394-2399.

Buttermann, G. R. and Mullin, W. J. (2008). Pain and disability correlated with disc degeneration via magnetic resonance imaging in scoliosis patients. Eur Spine J 17, 240249.

Carow, B. and Rottenberg, M. E. (2014). SOCS3, a Major Regulator of Infection and Inflammation. Front Immunol 5, 58.

Chen, C. H., Chiang, C. J., Wu, L. C., Yang, C. H., Kuo, Y. J., Tsuang, Y. H. and Tsai, T. H. (2016). Time course investigation of intervertebral disc degeneration in a rat-tail puncture model. Life Sci 156, 15-20.

Cortes, D. H. and Elliott, D. M. (2014). The Intervertebral Disc: Overview of Disc Mechanics. In The Intervertebral Disc: Molecular and Structural Studies of the Disc in Health and Disease (ed. I. M. Shapiro \& M. V. Risbud), pp. 17-31. Vienna: Springer Vienna.

Dudli, S., Ferguson, S. J. and Haschtmann, D. (2014). Severity and pattern of post-traumatic intervertebral disc degeneration depend on the type of injury. Spine J 14, 1256-1264.

Dy, P., Wang, W., Bhattaram, P., Wang, Q., Wang, L., Ballock, R. T. and Lefebvre, V. (2012). Sox9 directs hypertrophic maturation and blocks osteoblast differentiation of growth plate chondrocytes. Dev Cell 22, 597-609.

El-Brolosy, M. A. and Stainier, D. Y. R. (2017). Genetic compensation: A phenomenon in search of mechanisms. PLoS Genet 13, e1006780. 
Erickson, G. R., Alexopoulos, L. G. and Guilak, F. (2001). Hyper-osmotic stress induces volume change and calcium transients in chondrocytes by transmembrane, phospholipid, and Gprotein pathways. J Biomech 34, 1527-1535.

Eskola, P. J., Kjaer, P., Sorensen, J. S., Okuloff, A., Wedderkopp, N., Daavittila, I., Ala-Kokko, L., Mannikko, M. and Karppinen, J. (2012). Gender difference in genetic association between IL1A variant and early lumbar disc degeneration: a three-year follow-up. Int J Mol Epidemiol Genet 3, 195-204.

Fardon, D. F., Williams, A. L., Dohring, E. J., Murtagh, F. R., Gabriel Rothman, S. L. and Sze, G. K. (2014). Lumbar disc nomenclature: version 2.0: Recommendations of the combined task forces of the North American Spine Society, the American Society of Spine Radiology and the American Society of Neuroradiology. Spine J 14, 2525-2545.

Fearing, B. V., Hernandez, P. A., Setton, L. A. and Chahine, N. O. (2018). Mechanotransduction and cell biomechanics of the intervertebral disc. JOR Spine 1.

Frazee, A. C., Pertea, G., Jaffe, A. E., Langmead, B., Salzberg, S. L. and Leek, J. T. (2015). Ballgown bridges the gap between transcriptome assembly and expression analysis. Nat Biotechnol 33, 243-246.

Garbers, C., Aparicio-Siegmund, S. and Rose-John, S. (2015). The IL-6/gp130/STAT3 signaling axis: recent advances towards specific inhibition. Curr Opin Immunol 34, 75-82.

Geng, F. S., Abbas, L., Baxendale, S., Holdsworth, C. J., Swanson, A. G., Slanchev, K., Hammerschmidt, M., Topczewski, J. and Whitfield, T. T. (2013). Semicircular canal morphogenesis in the zebrafish inner ear requires the function of gpr126 (lauscher), an adhesion class $\mathrm{G}$ protein-coupled receptor gene. Development 140, 4362-4374.

Hamann, J., Aust, G., Arac, D., Engel, F. B., Formstone, C., Fredriksson, R., Hall, R. A., Harty, B. L., Kirchhoff, C., Knapp, B., et al. (2015). International Union of Basic and Clinical Pharmacology. XCIV. Adhesion G protein-coupled receptors. Pharmacol Rev 67, 338-367.

Hayashi, S., Fujishiro, T., Hashimoto, S., Kanzaki, N., Chinzei, N., Kihara, S., Takayama, K., Matsumoto, T., Nishida, K., Kurosaka, M., et al. (2015). p21 deficiency is susceptible to osteoarthritis through STAT3 phosphorylation. Arthritis Res Ther 17, 314.

He, Y., Siebuhr, A. S., Brandt-Hansen, N. U., Wang, J., Su, D., Zheng, Q., Simonsen, O., Petersen, K. K., Arendt-Nielsen, L., Eskehave, T., et al. (2014). Type X collagen levels are elevated in serum from human osteoarthritis patients and associated with biomarkers of cartilage degradation and inflammation. BMC Musculoskelet Disord 15, 309.

Henry, S. P., Liang, S., Akdemir, K. C. and de Crombrugghe, B. (2012). The postnatal role of Sox9 in cartilage. J Bone Miner Res 27, 2511-2525.

Huang da, W., Sherman, B. T. and Lempicki, R. A. (2009). Bioinformatics enrichment tools: paths toward the comprehensive functional analysis of large gene lists. Nucleic Acids Res 37, 1-13.

Hunter, D. J., Nevitt, M., Losina, E. and Kraus, V. (2014). Biomarkers for osteoarthritis: current position and steps towards further validation. Best Pract Res Clin Rheumatol 28, 61-71.

Kadow, T., Sowa, G., Vo, N. and Kang, J. D. (2015). Molecular basis of intervertebral disc degeneration and herniations: what are the important translational questions? Clin Orthop Relat Res 473, 1903-1912.

Kalb, S., Martirosyan, N. L., Kalani, M. Y., Broc, G. G. and Theodore, N. (2012). Genetics of the degenerated intervertebral disc. World Neurosurg 77, 491-501. 
Kapoor, M., Martel-Pelletier, J., Lajeunesse, D., Pelletier, J. P. and Fahmi, H. (2011). Role of proinflammatory cytokines in the pathophysiology of osteoarthritis. Nat Rev Rheumatol 7, 33-42.

Karner, C. M., Long, F., Solnica-Krezel, L., Monk, K. R. and Gray, R. S. (2015). Gpr126/Adgrg6 deletion in cartilage models idiopathic scoliosis and pectus excavatum in mice. Hum Mol Genet 24, 4365-4373.

Kim, D., Langmead, B. and Salzberg, S. L. (2015). HISAT: a fast spliced aligner with low memory requirements. Nature Methods 12, 357.

Kitaoka, E., Satomura, K., Hayashi, E., Yamanouchi, K., Tobiume, S., Kume, K., Obinata, M. and Nagayama, M. (2001). Establishment and characterization of chondrocyte cell lines from the costal cartilage of SV40 large T antigen transgenic mice. J Cell Biochem 81, 571-582.

Kizawa, H., Kou, I., lida, A., Sudo, A., Miyamoto, Y., Fukuda, A., Mabuchi, A., Kotani, A., Kawakami, A., Yamamoto, S., et al. (2005). An aspartic acid repeat polymorphism in asporin inhibits chondrogenesis and increases susceptibility to osteoarthritis. Nat Genet 37, 138-144.

Kondo, M., Yamaoka, K., Sakata, K., Sonomoto, K., Lin, L., Nakano, K. and Tanaka, Y. (2015). Contribution of the Interleukin-6/STAT-3 Signaling Pathway to Chondrogenic Differentiation of Human Mesenchymal Stem Cells. Arthritis Rheumatol 67, 1250-1260.

Latourte, A., Cherifi, C., Maillet, J., Ea, H. K., Bouaziz, W., Funck-Brentano, T., Cohen-Solal, M., Hay, E. and Richette, P. (2017). Systemic inhibition of IL-6/Stat3 signalling protects against experimental osteoarthritis. Ann Rheum Dis 76, 748-755.

Liebscher, I., Schon, J., Petersen, S. C., Fischer, L., Auerbach, N., Demberg, L. M., Mogha, A., Coster, M., Simon, K. U., Rothemund, S., et al. (2014). A tethered agonist within the ectodomain activates the adhesion G protein-coupled receptors GPR126 and GPR133. Cell Rep 9, 2018-2026.

Lin, K. H. and Tang, S. Y. (2017). The Quantitative Structural and Compositional Analyses of Degenerating Intervertebral Discs Using Magnetic Resonance Imaging and ContrastEnhanced Micro-Computed Tomography. Ann Biomed Eng 45, 2626-2634.

Lin, K. H., Wu, Q., Leib, D. J. and Tang, S. Y. (2016). A novel technique for the contrastenhanced microCT imaging of murine intervertebral discs. J Mech Behav Biomed Mater 63, 66-74.

Liu, J. W., Abraham, A. C. and Tang, S. Y. (2015a). The high-throughput phenotyping of the viscoelastic behavior of whole mouse intervertebral discs using a novel method of dynamic mechanical testing. J Biomech 48, 2189-2194.

Liu, Z., Chen, J., Mirando, A. J., Wang, C., Zuscik, M. J., O'Keefe, R. J. and Hilton, M. J. (2015b). A dual role for NOTCH signaling in joint cartilage maintenance and osteoarthritis. Sci Signal 8, ra71.

Livshits, G., Zhai, G., Hart, D. J., Kato, B. S., Wang, H., Williams, F. M. and Spector, T. D. (2009). Interleukin-6 is a significant predictor of radiographic knee osteoarthritis: The Chingford Study. Arthritis Rheum 60, 2037-2045.

Long, F., Zhang, X. M., Karp, S., Yang, Y. and McMahon, A. P. (2001). Genetic manipulation of hedgehog signaling in the endochondral skeleton reveals a direct role in the regulation of chondrocyte proliferation. Development 128, 5099-5108. 
Mahr, S., Burmester, G. R., Hilke, D., Gobel, U., Grutzkau, A., Haupl, T., Hauschild, M., Koczan, D., Krenn, V., Neidel, J., et al. (2006). Cis- and trans-acting gene regulation is associated with osteoarthritis. Am J Hum Genet 78, 793-803.

Martin, M. (2011). Cutadapt removes adapter sequences from high-throughput sequencing reads. 2011 17, 3 \%J EMBnet.journal.

Matta, C., Zakany, R. and Mobasheri, A. (2015). Voltage-dependent calcium channels in chondrocytes: roles in health and disease. Curr Rheumatol Rep 17, 43.

Mogha, A., Benesh, A. E., Patra, C., Engel, F. B., Schoneberg, T., Liebscher, I. and Monk, K. R. (2013). Gpr126 functions in Schwann cells to control differentiation and myelination via G-protein activation. J Neurosci 33, 17976-17985.

Munir, S., Rade, M., Maatta, J. H., Freidin, M. B. and Williams, F. M. K. (2018). Intervertebral Disc Biology: Genetic Basis of Disc Degeneration. Curr Mol Biol Rep 4, 143-150.

Nguyen, Q. T., Jacobsen, T. D. and Chahine, N. O. (2017). Effects of Inflammation on Multiscale Biomechanical Properties of Cartilaginous Cells and Tissues. ACS Biomater Sci Eng 3, 2644-2656.

Osuka, K., Usuda, N., Aoyama, M., Yamahata, H., Takeuchi, M., Yasuda, M. and Takayasu, M. (2014). Expression of the JAK/STAT3/SOCS3 signaling pathway in herniated lumbar discs. Neurosci Lett 569, 55-58.

Patra, C., van Amerongen, M. J., Ghosh, S., Ricciardi, F., Sajjad, A., Novoyatleva, T., Mogha, A., Monk, K. R., Muhlfeld, C. and Engel, F. B. (2013). Organ-specific function of adhesion G protein-coupled receptor GPR126 is domain-dependent. Proc Natl Acad Sci U S A 110, 16898-16903.

Pertea, M., Pertea, G. M., Antonescu, C. M., Chang, T.-C., Mendell, J. T. and Salzberg, S. L. (2015). StringTie enables improved reconstruction of a transcriptome from RNA-seq reads. Nature Biotechnology 33, 290.

Sabiston, P., Adams, M. E. and Ho, Y. A. (1985). Automation of 1,9-dimethylmethylene blue dye-binding assay for sulfated glycosaminoglycans with application to cartilage microcultures. Anal Biochem 149, 543-548.

Sambrook, P. N., MacGregor, A. J. and Spector, T. D. (1999). Genetic influences on cervical and lumbar disc degeneration: a magnetic resonance imaging study in twins. Arthritis Rheum 42, 366-372.

Schust, J., Sperl, B., Hollis, A., Mayer, T. U. and Berg, T. (2006). Stattic: a small-molecule inhibitor of STAT3 activation and dimerization. Chem Biol 13, 1235-1242.

Smith, L. J., Nerurkar, N. L., Choi, K. S., Harfe, B. D. and Elliott, D. M. (2011). Degeneration and regeneration of the intervertebral disc: lessons from development. Dis Model Mech 4, 31-41.

Snelling, S. J., Davidson, R. K., Swingler, T. E., Le, L. T., Barter, M. J., Culley, K. L., Price, A., Carr, A. J. and Clark, I. M. (2016). Dickkopf-3 is upregulated in osteoarthritis and has a chondroprotective role. Osteoarthritis Cartilage 24, 883-891.

Soriano, P. (1999). Generalized lacZ expression with the ROSA26 Cre reporter strain. Nat Genet 21, 70-71.

Stannus, O., Jones, G., Cicuttini, F., Parameswaran, V., Quinn, S., Burgess, J. and Ding, C. (2010). Circulating levels of IL-6 and TNF-alpha are associated with knee radiographic 
osteoarthritis and knee cartilage loss in older adults. Osteoarthritis Cartilage 18, 14411447.

Sun, D., Liu, P., Cheng, J., Ma, Z., Liu, J. and Qin, T. J. B. M. D. (2017). Correlation between intervertebral disc degeneration, paraspinal muscle atrophy, and lumbar facet joints degeneration in patients with lumbar disc herniation. 18, 167.

Suzuki, S., Fujita, N., Fujii, T., Watanabe, K., Yagi, M., Tsuji, T., Ishii, K., Miyamoto, T., Horiuchi, K., Nakamura, M., et al. (2017). Potential Involvement of the IL-6/JAK/STAT3 Pathway in the Pathogenesis of Intervertebral Disc Degeneration. Spine (Phila Pa 1976) 42, E817E824.

Tonge, D. P., Pearson, M. J. and Jones, S. W. (2014). The hallmarks of osteoarthritis and the potential to develop personalised disease-modifying pharmacological therapeutics. Osteoarthritis Cartilage 22, 609-621.

Urban, J. P., Smith, S. and Fairbank, J. C. (2004). Nutrition of the intervertebral disc. Spine (Phila Pa 1976) 29, 2700-2709.

Vergroesen, P. P., Kingma, I., Emanuel, K. S., Hoogendoorn, R. J., Welting, T. J., van Royen, B. J., van Dieen, J. H. and Smit, T. H. (2015). Mechanics and biology in intervertebral disc degeneration: a vicious circle. Osteoarthritis Cartilage 23, 1057-1070.

Wilke, H. J., Kienle, A., Maile, S., Rasche, V. and Berger-Roscher, N. (2016). A new dynamic six degrees of freedom disc-loading simulator allows to provoke disc damage and herniation. Eur Spine J 25, 1363-1372.

Woessner, J. F., Jr. (1961). The determination of hydroxyproline in tissue and protein samples containing small proportions of this imino acid. Arch Biochem Biophys 93, 440-447.

Wuertz, K. and Haglund, L. (2013). Inflammatory mediators in intervertebral disk degeneration and discogenic pain. Global Spine J 3, 175-184.

Yao, Y. and Wang, Y. (2013). ATDC5: an excellent in vitro model cell line for skeletal development. J Cell Biochem 114, 1223-1229.

Yee, A. and Chan, D. (2014). Genetic Basis of Intervertebral Disc Degeneration. In The Intervertebral Disc: Molecular and Structural Studies of the Disc in Health and Disease (ed. I. M. Shapiro \& M. V. Risbud), pp. 157-176. Vienna: Springer Vienna.

Yee, A., Lam, M. P., Tam, V., Chan, W. C., Chu, I. K., Cheah, K. S., Cheung, K. M. and Chan, D. (2016). Fibrotic-like changes in degenerate human intervertebral discs revealed by quantitative proteomic analysis. Osteoarthritis Cartilage 24, 503-513.

Zhang, Q., Lin, S., Liu, Y., Yuan, B., Harris, S. E. and Feng, J. Q. (2016). Dmp1 Null Mice Develop a Unique Osteoarthritis-like Phenotype. Int J Biol Sci 12, 1203-1212.

Zhang, Y., Xiong, C., Kudelko, M., Li, Y., Wang, C., Wong, Y. L., Tam, V., Rai, M. F., Cheverud, J., Lawson, H. A., et al. (2018). Early onset of disc degeneration in SM/J mice is associated with changes in ion transport systems and fibrotic events. Matrix Biol 70, 123-139.

Zhao, C. Q., Wang, L. M., Jiang, L. S. and Dai, L. Y. (2007). The cell biology of intervertebral disc aging and degeneration. Ageing Res Rev 6, 247-261.

Zhu, F., Bao, H., Yan, P., Liu, S., Bao, M., Zhu, Z., Liu, Z. and Qiu, Y. (2017a). Do the disc degeneration and osteophyte contribute to the curve rigidity of degenerative scoliosis? BMC Musculoskeletal Disorders 18, 128. 
bioRxiv preprint doi: https://doi.org/10.1101/581595; this version posted March 18, 2019. The copyright holder for this preprint (which was not certified by peer review) is the author/funder, who has granted bioRxiv a license to display the preprint in perpetuity. It is made available under aCC-BY 4.0 International license.

Zhu, F., Bao, H., Yan, P., Liu, S., Bao, M., Zhu, Z., Liu, Z. and Qiu, Y. J. B. M. D. (2017b). Do the disc degeneration and osteophyte contribute to the curve rigidity of degenerative scoliosis? 18, 128. 


\section{Figures and figure legends}
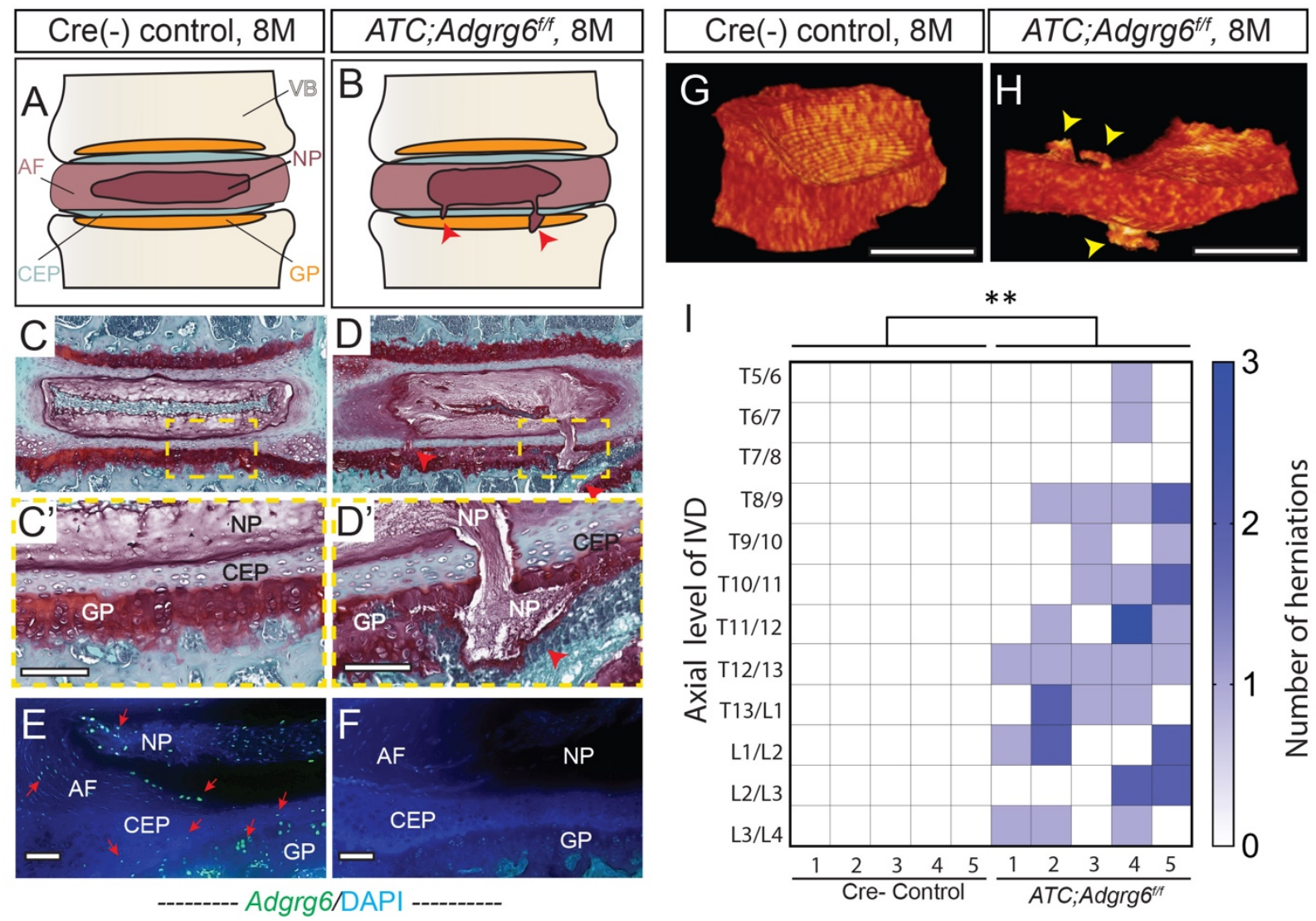

Figure 1: Adult $\boldsymbol{A T C}$; Adgrg $^{f / f}$ mutant mice display endplate-oriented herniations of the IVD. (A and B) Schematic of endplate-oriented herniations (B, red arrowheads) observed in $A T C$; $A d g r g 6^{f / f}$ mutant mice (B), in contrast to a typical wild-type IVD (A) at 8 months of age. (C-D') Representative medial-sectioned 8-month-old mouse IVDs stained with Safranin-O/Fast green ( $\mathrm{SO} / \mathrm{FG})$ ( $n=3$ for each group). (E, F) Adgrg6 riboprobe FISH (green fluorescence) at 8 months. $(\mathrm{G}, \mathrm{H})$ Representative reconstructions of contrast-enhanced $\mu \mathrm{CT}$ of Cre (-) control $(\mathrm{G})$ and $A T C ; A d g r g f / f$ mutant $(\mathrm{H})$ IVDs at 8 months of age. Endplate-oriented herniations are observed in SO/FG stained sections (D', red arrowheads) and by contrast-enhanced $\mu \mathrm{CT}(\mathrm{H}$, yellow arrowheads) ( $n=5$ for each group). (I) Heat map of contrast-enhanced $\mu C T$ data from five Cre (-) control and five ATC;Adgrgfff mutant mouse spines, plotting the axial level of the IVD (left axis) and the number of herniations (right axis) observed in each mouse. $(* * p \leq 0.01$, Student's $t$ Test.) Scale bars: $50 \mu \mathrm{m}$ in (C', D'), $100 \mu \mathrm{m}$ in (E, F), and $500 \mu \mathrm{m}$ in $(\mathrm{G}, \mathrm{H}) . A F-$ annulus fibrosis, CEP-cartilaginous endplate, GP-growth plate, NP-nucleus pulposus, and VBvertebral body. 

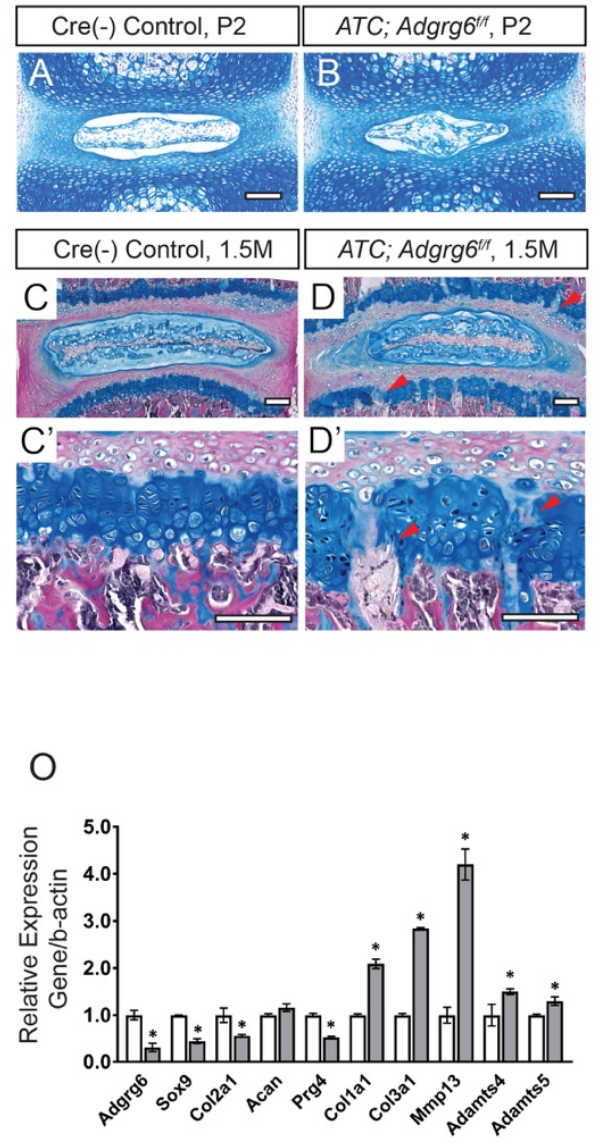

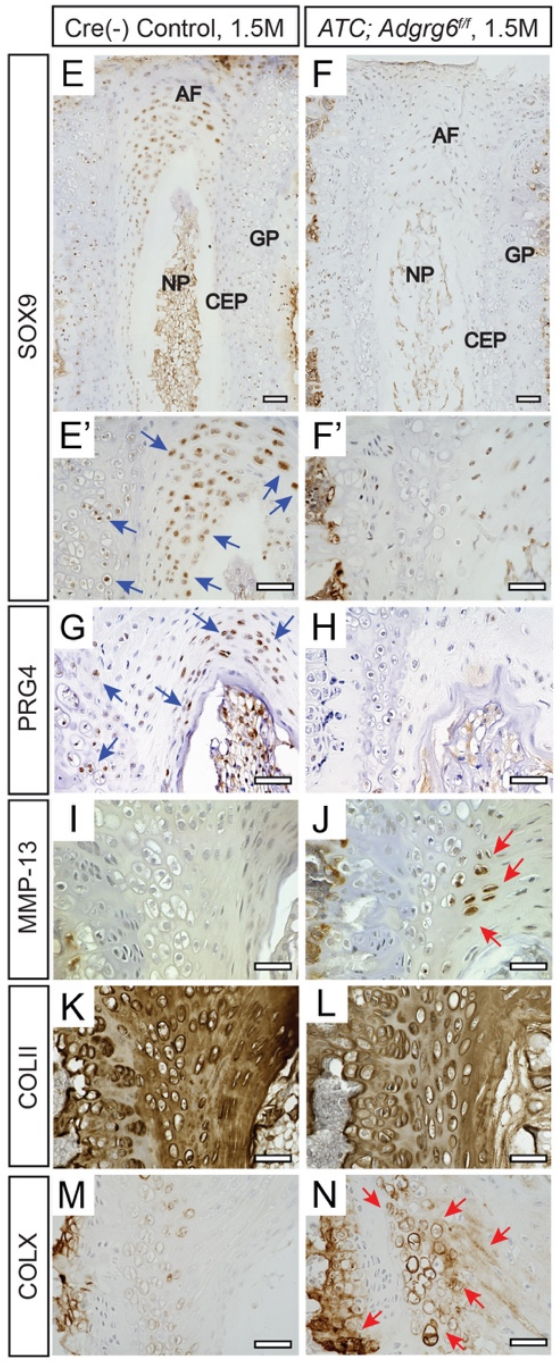

Figure 2: Young $A T C$; $A d g r g 6^{f / f}$ mutant mice display alterations in IVDs consistent with disc degeneration pathology. (A-B') Representative medial-sectioned IVD tissues stained with Alcian blue-Hematoxylin/Orange G. ATC;Adgrgfff mutant and control IVDs are comparable at P2 (A, B) and 1.5 months (C-D') of age, except for the mild increase of acellular clefts in the GP at 1.5 months (red arrowheads, D, D' $n=3$ for each group.) (E-O) IHC (E-N) and qPCR (O) analyses of common markers of degenerative disc in mice at 1.5 months. ATC;Adgrgf/f mutant IVDs display reduced expression of markers of healthy disc: SOX9/Sox 9 (blue arrows, E') and PRG4/Prg4 (blue arrows, G), and mildly reduced COLII/Col2al (K). They also display increased expression of hypertrophic marker COLX/Colloal (red arrows, N), extracellular matrix modifying enzymes (MMP-13/Mmp13 (red arrows, J), Adamts4, and Adamts5), dedifferentiation marker (Collal), and fibrosis marker (Col3al). (C-N, n= 3 for each group. O, $n=$ 3 biological replicates. Bars represent mean $\pm \mathrm{SD}$. ${ }^{*} \mathrm{p} \leq 0.05$, Student's $t$ Test.) Scale bars: $100 \mu \mathrm{m}$ in (A, B) and (C', D'); $200 \mu \mathrm{m}$ in (C, D); $50 \mu \mathrm{m}$ in (E-N). AF-annulus fibrosis, CEPcartilaginous endplate, GP-growth plate, and NP-nucleus pulposus. 


\section{5 months}
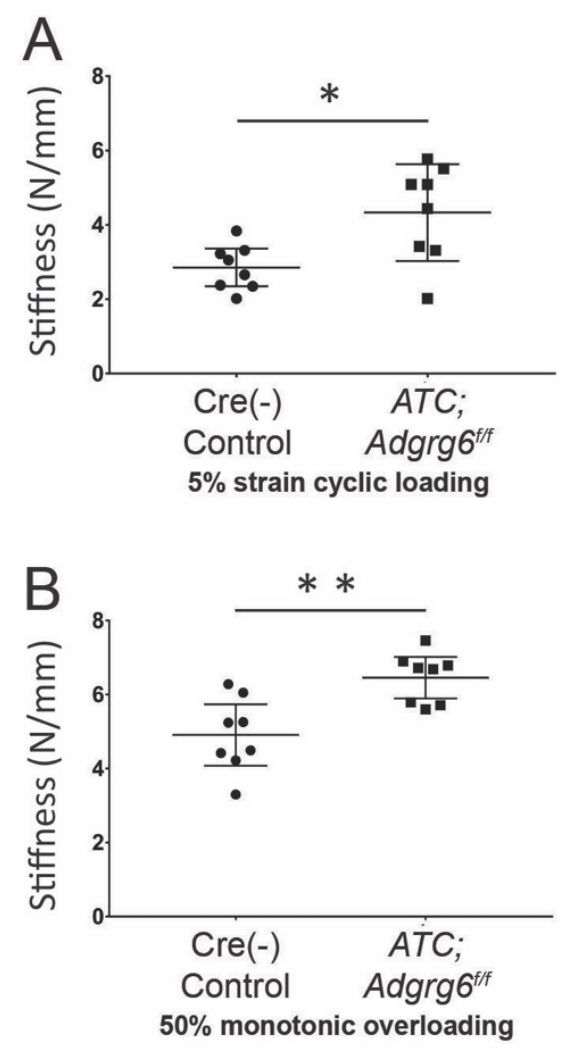

Figure 3: Young ATC;Adgrg fff $^{f f}$ mutant mice display increased mechanical stiffness of the IVD. (A, B) Mechanical testing using 5\% strain cyclic loading (stiffness mean w/95\% CI, p < 0.05 ) (A), and 50\% monotonic overloading (stiffness mean w/95\% CI, p < 0.01) (B), demonstrating increased stiffness in ATC;Adgrgfff mutant lumbar IVDs. ( $n=4$ for each group, 4 IVDs were analyzed /mouse.) 


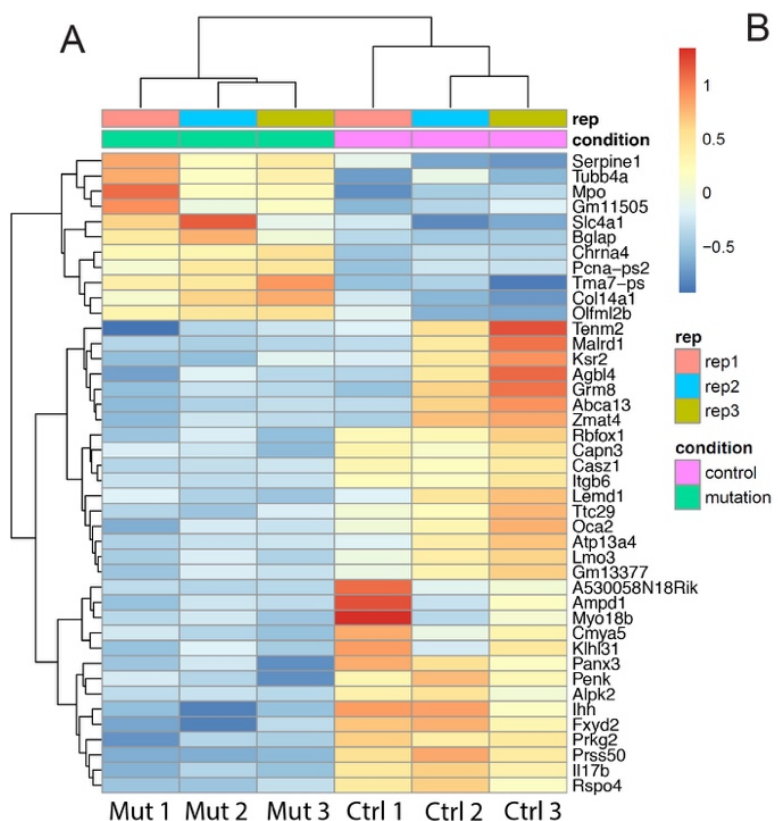

C

\begin{tabular}{|r|r|}
\multicolumn{2}{|c}{ Chondrogenic } \\
\hline Gene & Log2 FC \\
\hline ox5 & -0.47 \\
\hline ox6 & -0.61 \\
\hline can & 0.09 \\
\hline col2a1 & -0.52 \\
\hline ol9a2 & -0.4 \\
\hline col9a3 & 0.27 \\
\hline ol11a1 & -0.71 \\
\hline fofr3 & -0.76 \\
\hline
\end{tabular}

D

Catabolic

\begin{tabular}{|l|r|}
\hline \multicolumn{1}{|c|}{ Gene } & Log2 FC \\
\hline Mmp2 & 0.43 \\
\hline Mmp3 & 0.81 \\
\hline Mmp9 & 0.05 \\
\hline Mmp11 & 0.33 \\
\hline Mmp13 & -0.33 \\
\hline Mmp14 & 0.02 \\
\hline Mmp15 & 0.71 \\
\hline Adamts2 & 0.74 \\
\hline Adamts4 & 0.44 \\
\hline Adamts5 & 0.23
\end{tabular}

B

\begin{tabular}{|c|c|c|}
\hline \multicolumn{3}{|c|}{ Enriched GO Terms } \\
\hline ID & Description & $P$ value \\
\hline GO:0005615 & extracellular space & 2.71E-09 \\
\hline GO:0005576 & extracellular region & $6.49 \mathrm{E}-09$ \\
\hline GO:0031012 & extracellular matrix & $1.84 \mathrm{E}-07$ \\
\hline GO:0048146 & $\begin{array}{l}\text { positive regulation of } \\
\text { fibroblast proliferation }\end{array}$ & 8.78E-07 \\
\hline GO:0062023 & $\begin{array}{l}\text { collagen-containing } \\
\text { extracellular matrix }\end{array}$ & 7.31E-06 \\
\hline GO:0005201 & $\begin{array}{l}\text { extracellular matrix } \\
\text { structural constituent }\end{array}$ & 4.40E-05 \\
\hline GO:0030020 & $\begin{array}{l}\text { extracellular matrix } \\
\text { structural constituent } \\
\text { conferring tensile strength }\end{array}$ & $7.32 \mathrm{E}-05$ \\
\hline GO:0043062 & $\begin{array}{l}\text { extracellular structure } \\
\text { organization }\end{array}$ & $8.62 \mathrm{E}-05$ \\
\hline GO:1901681 & sulfur compound binding & $6.13 E-04$ \\
\hline GO:0042509 & $\begin{array}{l}\text { regulation of tyrosine } \\
\text { phosphorylation of STAT } \\
\text { protein }\end{array}$ & $8.91 \mathrm{E}-04$ \\
\hline GO:0006811 & ion transport & $1.37 \mathrm{E}-02$ \\
\hline GO:0042626 & $\begin{array}{l}\text { ATPase activity, coupled to } \\
\text { transmembrane }\end{array}$ & $2.22 \mathrm{E}-02$ \\
\hline GO:0019722 & $\begin{array}{l}\text { calcium-mediated } \\
\text { signaling }\end{array}$ & $2.92 \mathrm{E}-02$ \\
\hline
\end{tabular}

$\mathrm{E}$

\begin{tabular}{|l|r|}
\multicolumn{2}{|c}{ Fibrous collagens } \\
\hline Gene & Log2 FC \\
\hline Col1a1 & 0.79 \\
\hline Col1a2 & 0.96 \\
\hline Col3a1 & 1.21 \\
\hline Col4a1 & 0.43 \\
\hline Col4a2 & 0.48 \\
\hline Col5a1 & 0.81 \\
\hline Col5a2 & 0.95 \\
\hline Col5a3 & 0.48 \\
\hline Col14a1 & 1.25 \\
\hline Col15a1 & 0.73 \\
\hline
\end{tabular}

$\mathrm{F}$

Ion transport

\begin{tabular}{|c|c|}
\hline Gene & Log2 FC \\
\hline Fxyd2 & -1.15 \\
\hline Capn3 & -1.07 \\
\hline Panx3 & -1.05 \\
\hline Atp13a4 & -1 \\
\hline Serpine1 & 1.08 \\
\hline Chrna4 & 1.4 \\
\hline Slc4a1 & 1.46 \\
\hline \multicolumn{2}{|c|}{ G } \\
Suppressor of cytokine \\
signaling \\
\hline Gene & Log2 FC \\
\hline Socs1 & 0.65 \\
Socs3 & 0.92 \\
\hline
\end{tabular}

Figure 4: Young Col2Cre;Adgrg6fff mutant mice display fibrotic-like changes of gene expression and dysregulation of genes associated with ion transport in the IVD. (A) Heatmap of differentially expressed gene based on RNA-sequencing analysis of IVDs derived from both Col2Cre;Adgrgfff mutant (Mut 1-3) and Cre (-) littermates (Ctrl 1-3) at P20. (B) Gene ontology (GO) analysis revealed a suite of differentially expressed genes important for extracellular matrix organization and ion transport. (C-G) RNA-sequencing analysis revealed mild alterations in some chondrogenic (C) and catabolic (D) gene expression, but significantly induced fibrotic gene expression (E) and dysregulation of genes involved in ion transport $(F)$ in the Col2Cre;Adgrgff/ mutant IVDs at P20. Some genes encode members of the suppressor of cytokine signaling were also upregulated in the mutant IVDs $(\mathrm{G})$. Differential expressed genes with $p$ value $<0.05$ were highlighted in blue. 

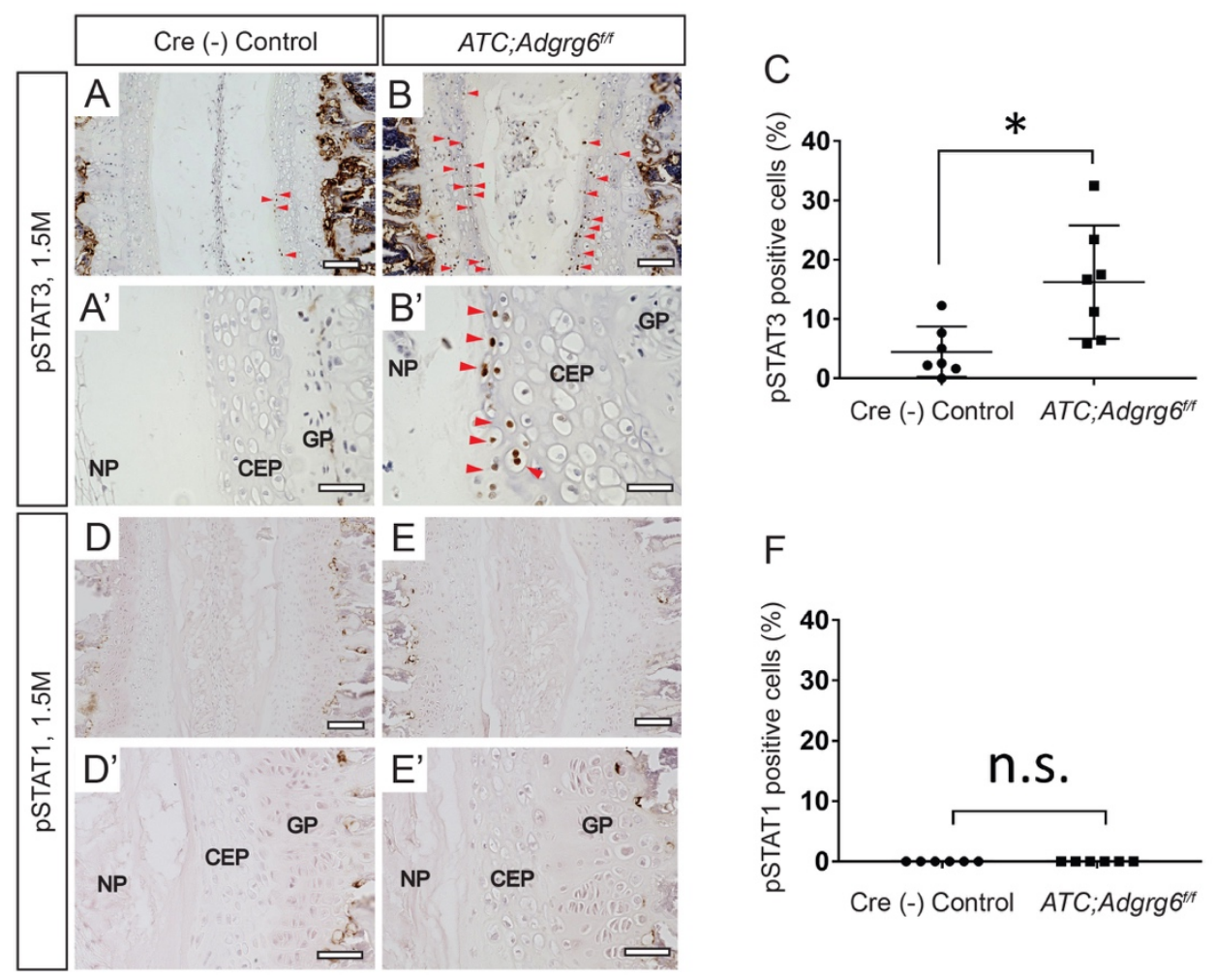

Figure 5: ADGRG6 regulates STAT3 signaling in IVDs. (A-B') IHC analysis shows increased expression of pSTAT3 (red arrowheads, B, B') in ATC; Adgrgf/f mutant mouse IVD at 1.5months ( $n=3$ for each group.) (C) Quantification of positive pSTAT3 cells in Cre (-) control or in $A T C$; $A d g r g^{f / f}$ mutant mouse IVDs. ( $n=3$ mice for each group, at least two IVDs scored for each mouse. Dots plot with mean \pm SD. ${ }^{*} \mathrm{p} \leq 0.05$, Student's $t$ Test.) Scale bars: $200 \mu \mathrm{m}$ in (A, B), and $50 \mu \mathrm{m}$ in (A', B'). CEP-cartilaginous endplate, GP-growth plate, and NP-nucleus pulposus. 

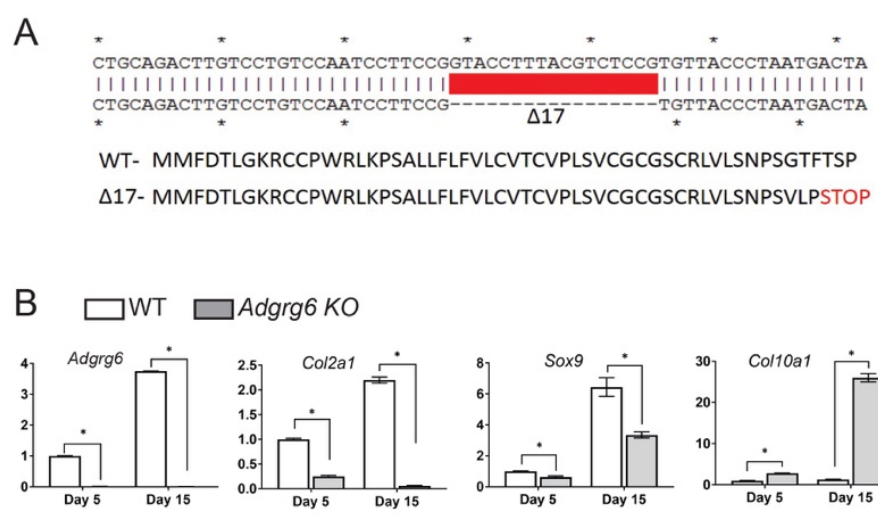

Adgrg6 KO
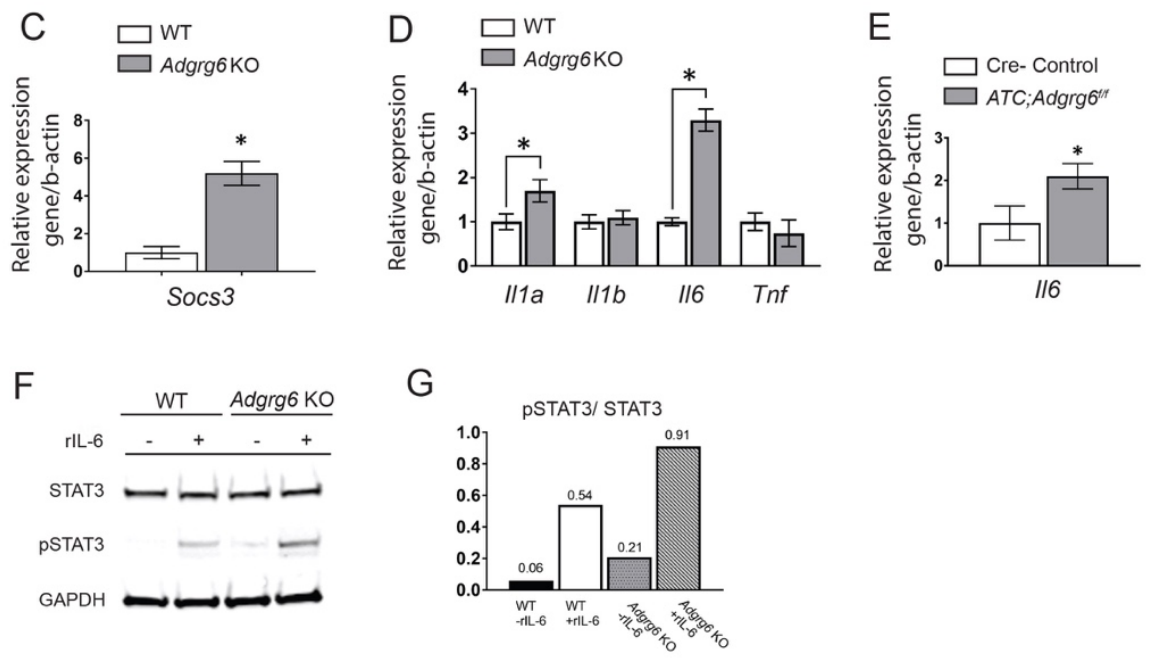

Figure 6: Adgrg6 regulates gene expression profiles and STAT3 signaling in ATDC5 cell culture. (A) Schematic of a 17-bp deletion of Adgrg6 from a stable single cell clone of ATDC5 cell line (Adgrg6 KO). (B) Bright field image of ATDC5 cells maturated for 5 days demonstrates hypertrophic morphology in Adrgr6 KO cells compared to wild type control cells. (C) qPCR analyses of gene expression in ATDC 5 cells at 5 and 15 days of maturation demonstrates decreased expression of markers of healthy disc, Col2aland Sox9, and increased expression of the hypertrophic marker, CollOal and the extracellular matrix modifying enzyme, Mmp 13 in Adgrg6 KO cells. (D) qPCR analyses revealed increased expression of Socs 3 in Adrgr6 KO cells after 15 days of maturation. (E) qPCR analysis of Illa, Illb, Il6 and Tnf in ATDC5 cells maturated for 15 days. (F) qPCR analysis of Il6 expression in 1.5-month-old primary mouse IVDs. (C-F, $n=3$ biological replicates and representative result is shown. Bars represent mean \pm SD. ${ }^{*} \mathrm{p} \leq 0.05$, Student's $t$ Test). (G, H) Representative Western blot and quantification of wild type and Adgrg6 KO ATDC5 cell lysates showing stimulation of pSTAT3 staining after treatment with recombinant IL-6 (rIL-6) protein in both cell lines, while Adgrg6 KO cells show a mild constitutive stimulation of pSTAT3 without addition of rIL-6 $(n=3$ biological replicates and representative result is shown). Scale bars: $50 \mu \mathrm{m}$ in (B). 

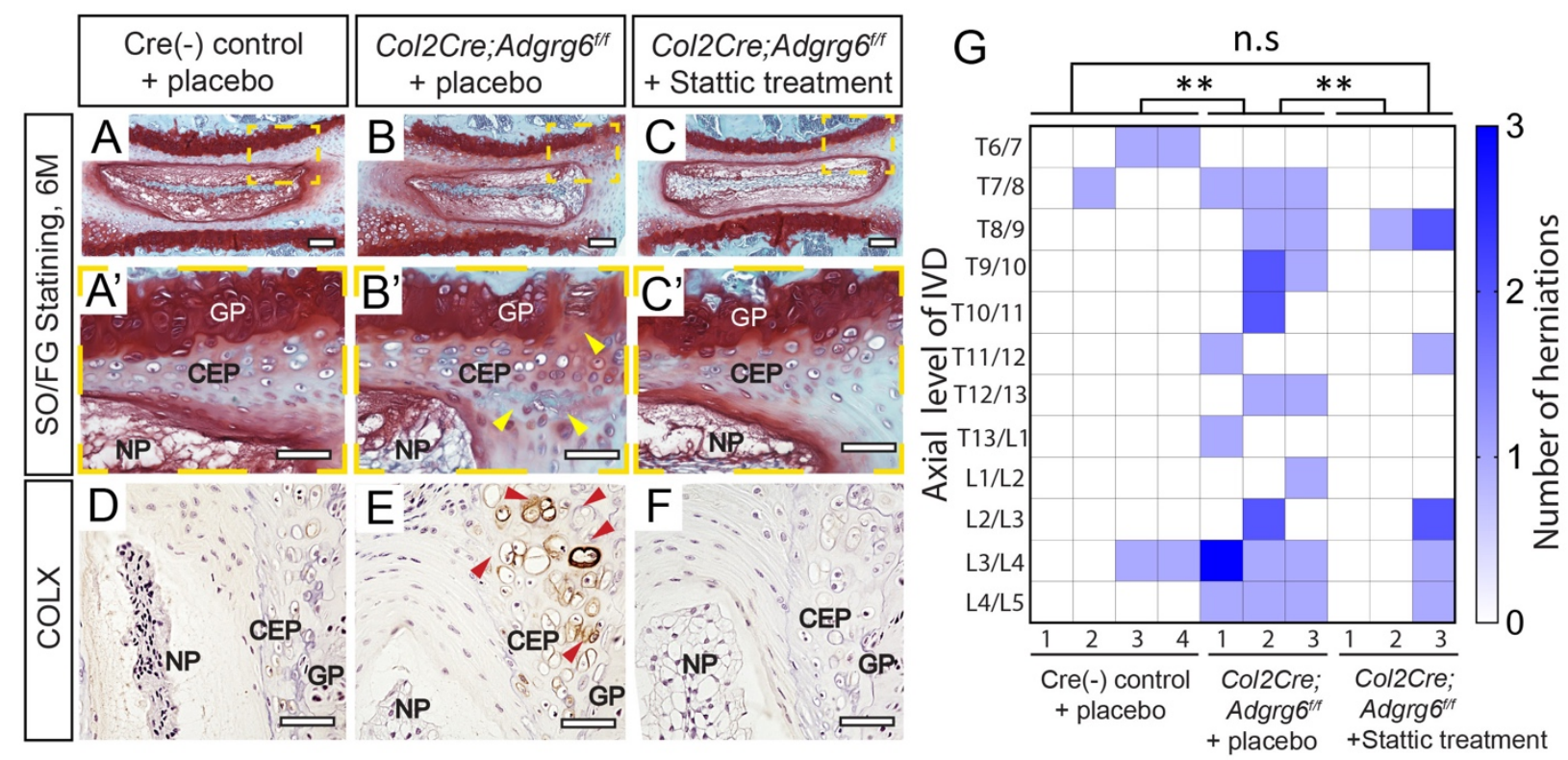

Figure 7: Inhibition of STAT3 by Stattic alleviates the formation of disc herniations attributed to loss of $\boldsymbol{A d g r g} 6$ function. (A-C') Representative Safranin-O/Fast green staining and (D-F) COLX IHC in medial-sectioned mouse IVD from placebo-treated Cre (-) control (AA', D), placebo-treated Col2Cre;Adgrg6fff mutant (B-B', E), and Stattic treated Col2Cre;Adgrg6f/f mutant mice (C-C', F). Col2Cre;Adgrg6 f/f mutant mice display defects of the IVD including lesions and clefts in the CEP and GP (yellow arrowheads, B') and increased, ectopic expression of COLX within the CEP (red arrowheads, E), which is reduced by Stattic treatment (C-C', F). (G) Heat map to represent contrast-enhanced microCT data from 6-monthold mice from three experimental groups: four placebo-treated Cre (-) controls; three placebotreated Col2Cre;Adgrgff/f mutants; and three Stattic-treated Col2Cre;Adgrgff/f mutants. Plotted by the axial level of the IVD (left axis) and the number of herniations (right axis) observed in each mouse. ( ${ }^{* *} \mathrm{p} \leq 0.01$, One way ANOVA followed by Tukey HSD test. n.s, not significant.) Scale bars: $200 \mu \mathrm{m}$ in (A-C), $50 \mu \mathrm{m}$ in (A'-C') and (D-F). CEP-cartilaginous endplate, GP-growth plate, and NP-nucleus pulposus. 


\section{Supplemental Figures}
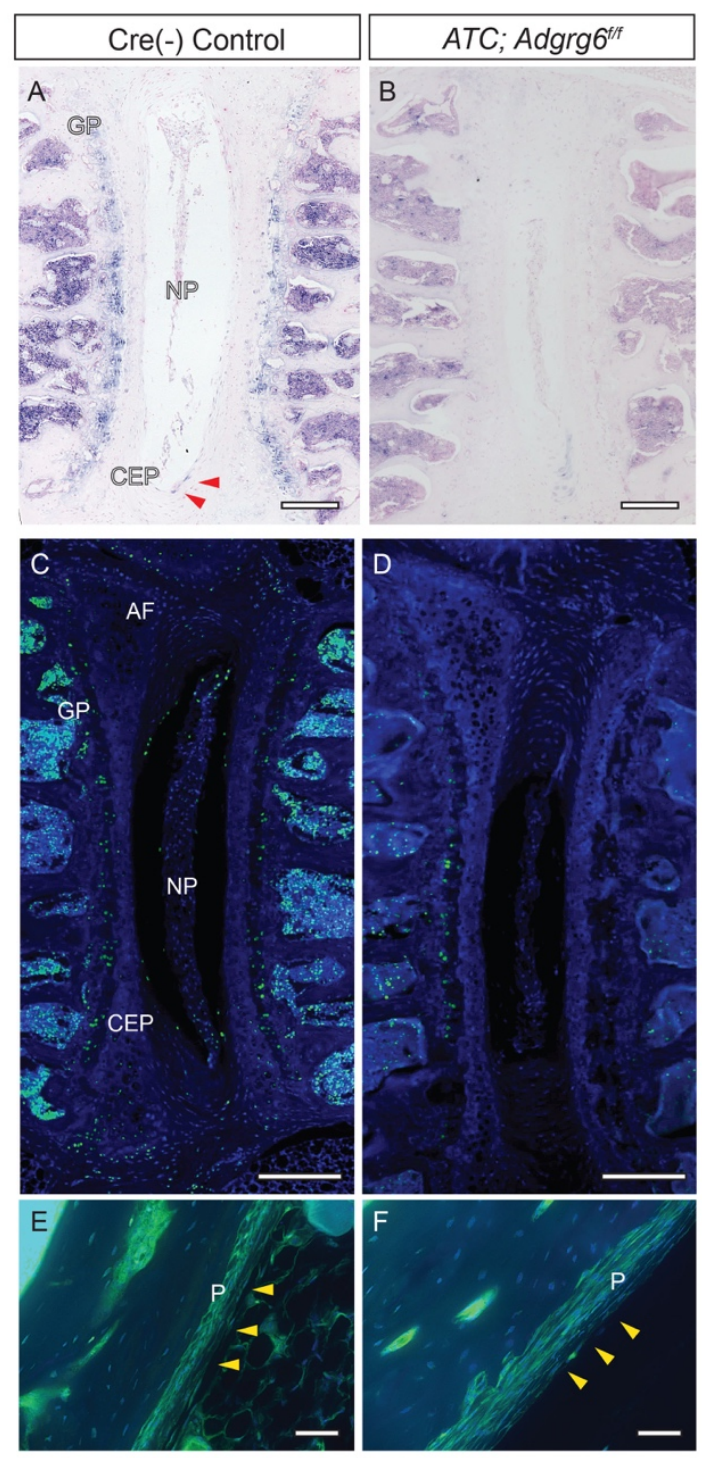

--o---Adgrg6/DAPI .

Supplemental Figure 1: In situ expression of Adgrg6 in the spine. (A, B) In situ hybridizations of Adgrg6 in spine tissue (8 months) using Alkaline phosphatase/BM purple chromogenic developing shows strong Adgrg6 expression (blue stain) in the growth plate (GP) and minor expression in the annulus fibrosis (AF) (red arrowheads) that is mostly abolished in $A T C$; $\operatorname{Adgrg} 6^{\text {fff }}$ mutant tissues (B); or using (C, D) tyramine-amplification fluorescence which shows expanded expression throughout the IVD including GP, CEP, AF, and NP, which is mostly diminished in $A T C ; A d g r g 6^{f f f}$ mutant tissues. Robust expression was detected in 
periosteum of the long bone tissues in both control and the $A T C ; A d g r g f^{f / f}$ mutant moues (E, F, yellow arrows). Scale bars: $200 \mu \mathrm{m}$ in (A-D); $50 \mu \mathrm{m}$ in (E, F). CEP-cartilaginous endplate, GP-
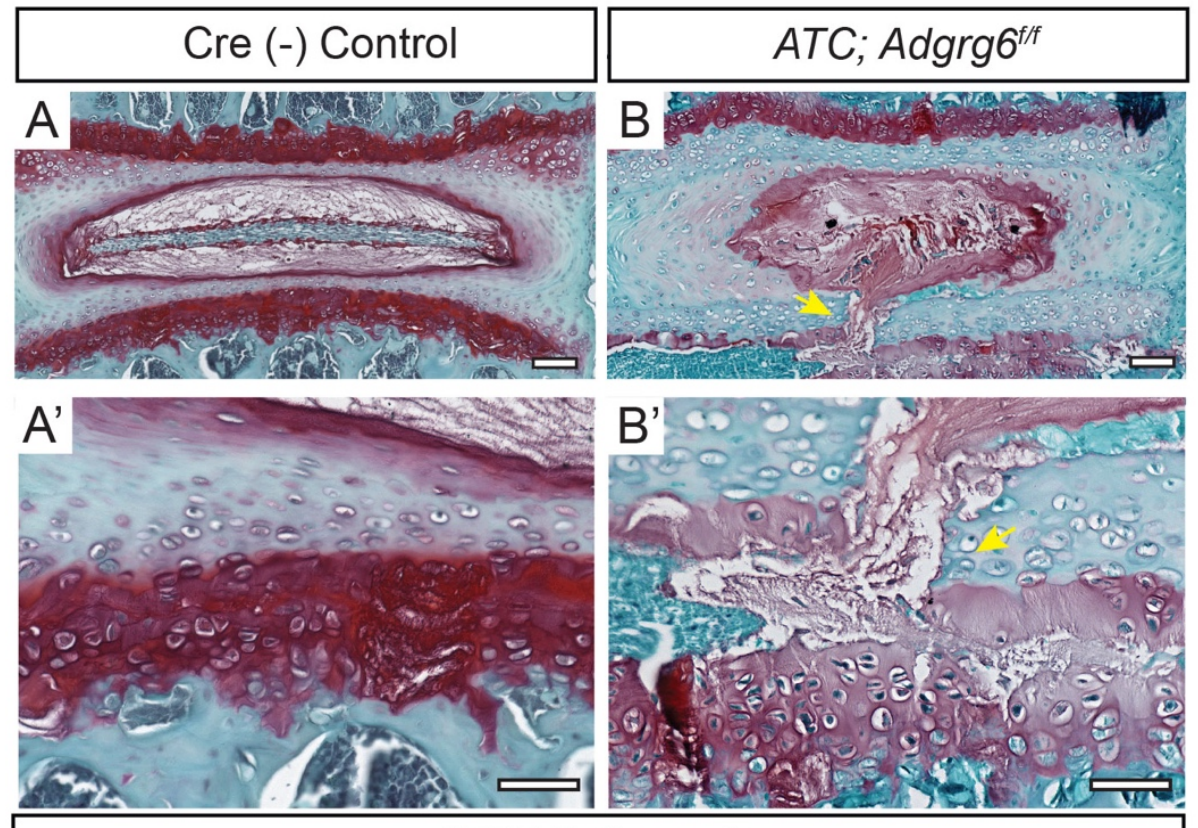

SO/FG, 6 months

Supplemental Figure 2: Embryonic loss of Adgrg6 lead to endplate-oriented herniations of the IVD in adult $\boldsymbol{A T C}$; Adgrg $^{\text {fff }}$ mutant mice. (A-B') Representative medial-sectioned 6month-old mouse IVDs (induced form E0.5-P20) stained with Safranin-O/Fast green (SO/FG) ( $n=3$ for controls and $n=6$ for mutants). Endplate-oriented disc herniation is indicated with yellow arrows. Scale bars: $100 \mu \mathrm{m}$ in (A, B); $50 \mu \mathrm{m}$ in (A', B'). 

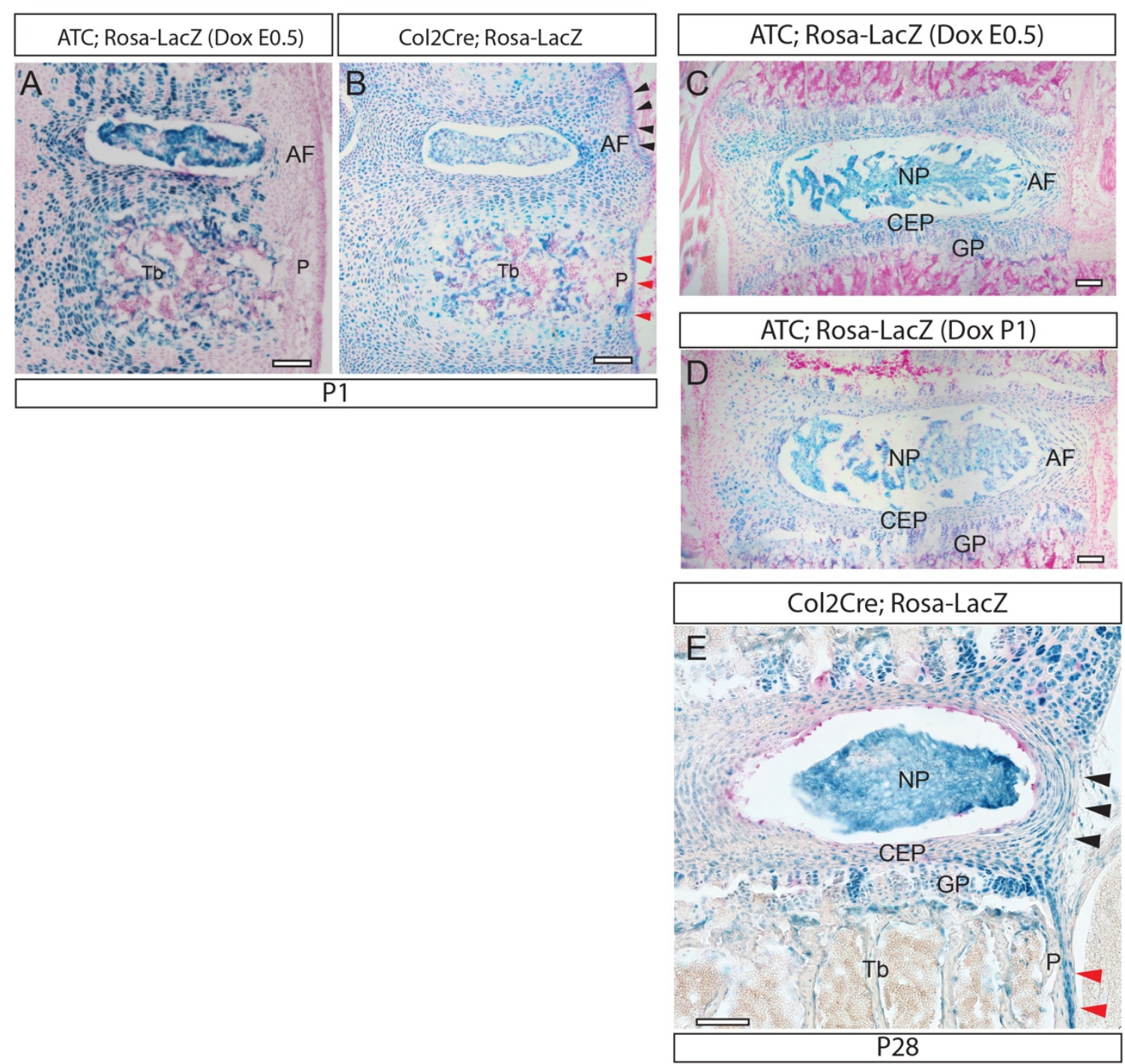

Supplemental Figure 3: $\beta$-galactosidase staining of LacZ-reporter mice induced with different strategies. More robust recombination (blue signal) in CEP, GP, and AF of the IVD was observed in the Col2Cre; Rosa-LacZ mouse (B, E) compared with the ATC; Rosa-LacZ mouse when induced from E0.5-P20 (A, C) and P1-P20 (D). Recombination in periosteum (B, E, red arrows) and the outmost AF layers of the IVD (B, E, black arrows) was observed only in the Col2Cre; Rosa-LacZ mouse but not the ATC; Rosa-LacZ mouse. Scale bars: $100 \mu \mathrm{m}$ in (A-E). $C E P$-cartilaginous endplate, GP-growth plate, AF-annulus fibrosis, NP-nucleus pulposus, Tbtrabecular bone, and $P$-periosteum. 

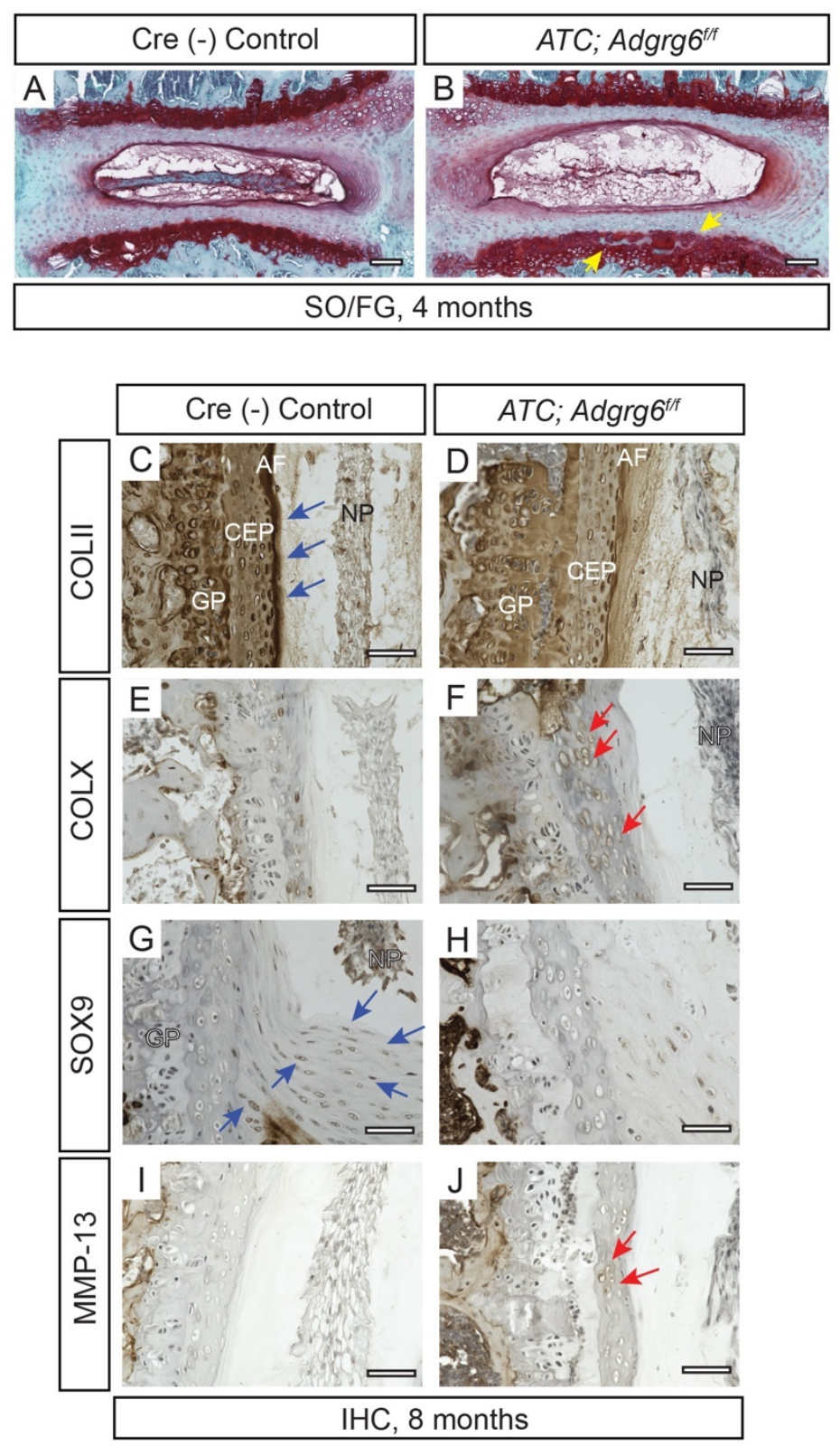

Supplemental Figure 4: Postnatal loss of $A d g r g 6$ in $A T C$; $A d g r g 6^{f / f}$ mutant mice leads to degenerative changes in the IVDs. (A, B) Representative medial-sectioned 4-month-old mouse IVDs stained with Safranin-O/Fast green (SO/FG) (induced from P1-P20, $n=3$ for controls and $n=5$ for mutants). Minor growth plate erosion is observed in two out of five mutant mice (B, yellow arrowheads). (C-J) IHC analysis of 8-month-old Cre (-) Control and ATC; Adgrgf/f mutant mouse IVDs (induced from P1-P20). Several protein markers of IVD health and disease are affected in $A T C ; A d g r g f / f$ mutant IVD including decreased expression of healthy disc markers COLII (C, blue arrows) and SOX9 (G, blue arrows), and increased expression of the hypertrophic marker COLX (F, red arrows) and extracellular matrix modifying enzyme MMP-13 (J, red arrows). ( $n=3$ for each group.) Scale bars: $100 \mu \mathrm{m}$ in (A, B); $50 \mu \mathrm{m}$ in (C-J). CEP: cartilaginous endplate, GP: growth plate, AF: annulus fibrosis, and NP: nucleus pulposus. 


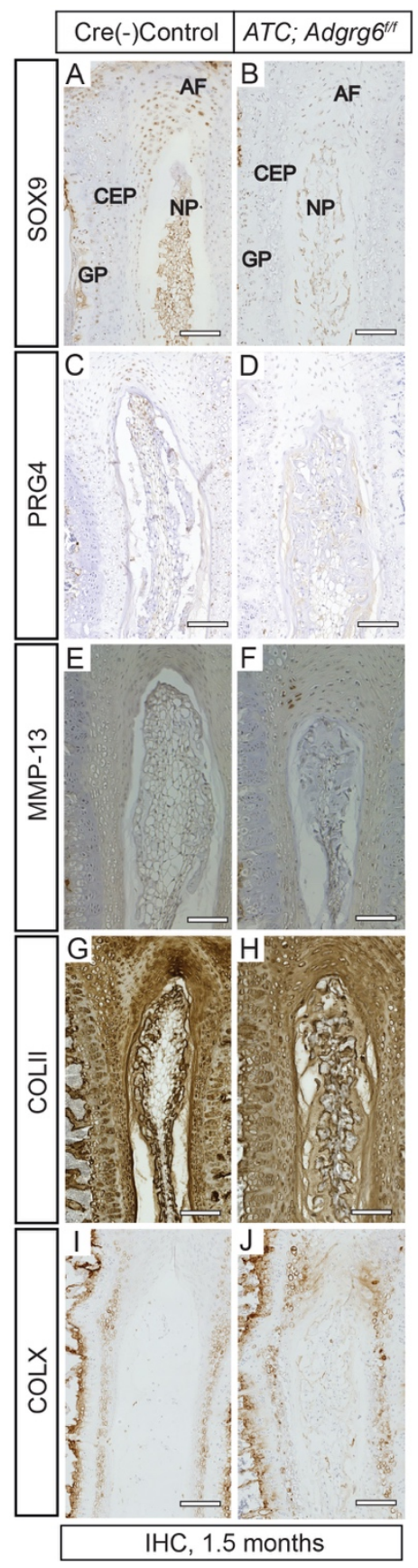

Supplemental Figure 5: Young $A T C$; $A$ dgrg $6^{f f f}$ mutant mice display degenerative alterations of protein expression in the IVD. Large scale images of IHC analysis shown in Figure 2. IHC analysis of common markers of degenerative disc. ATC;Adgrgfff conditional mutant IVDs display reduced expression of markers of healthy disc: SOX9 (B), PRG4 (D), and COLII (H); and increased expression of extracellular matrix modifying enzymes MMP-13 (F), hypertrophic marker COLX (J). Scale bars: $100 \mu \mathrm{m}$ in (A-J). AF- annulus fibrosis, CEP-cartilaginous endplate, GP-growth plate, and NP-nucleus pulposus. 

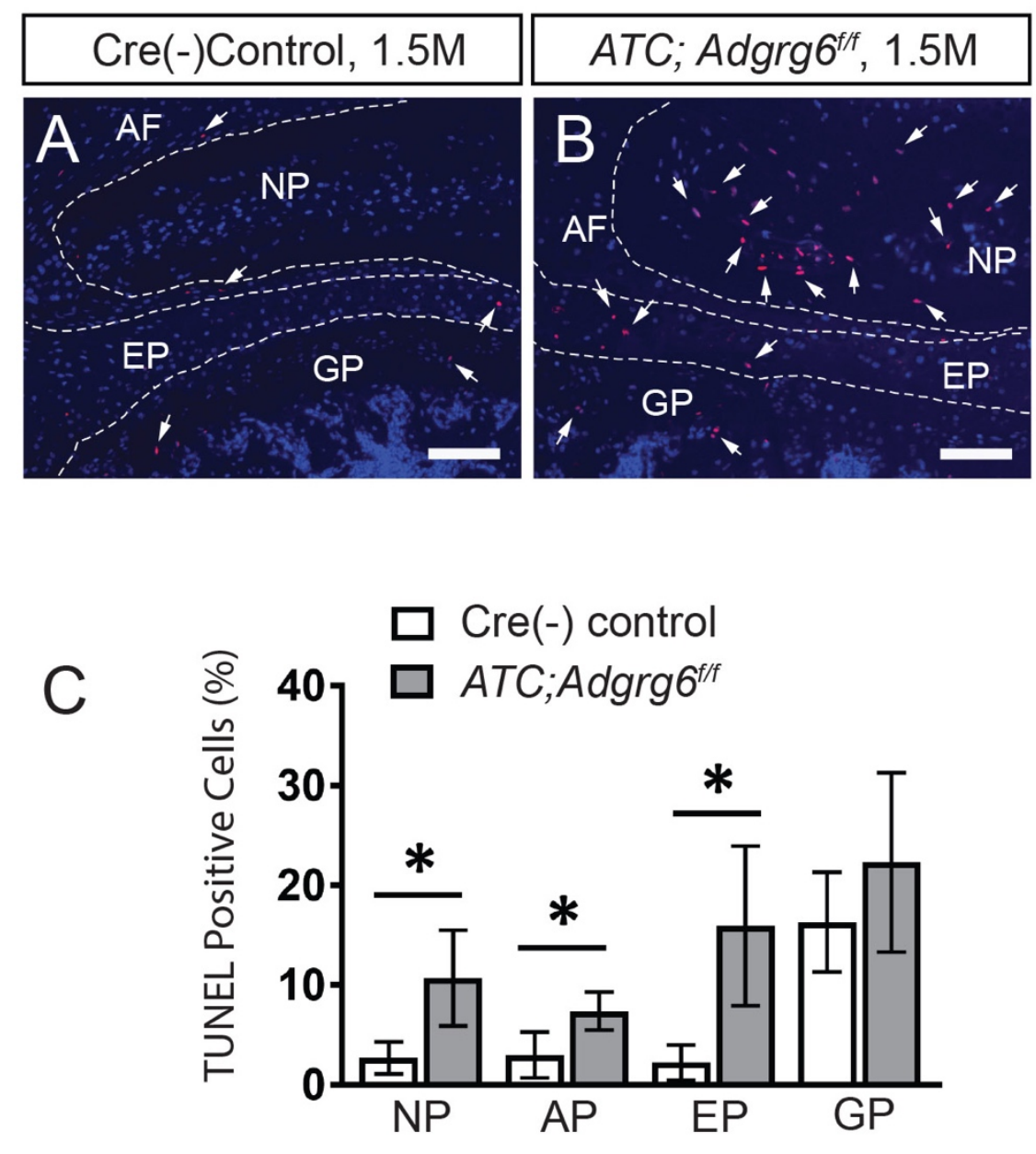

Supplemental Figure 6: Young $A T C$; $A d g r g 6^{f f f}$ conditional mutant mice display increased apoptosis in the IVD. (A, B) TUNEL (red fluorescence) staining of 1.5-month-old ATC; Adgrgfff mutants (B, white arrows) display increased TUNEL positive cells compared to Cre (-) control (A) mice. (C) Graph of the ratio of TUNEL positive cells to total cells (DAPI) ( $n=3$ for each group, 3-5 IVDs were analyzed/mouse. Bars represent mean \pm SD. * $\mathrm{p} \leq 0.05$, Student's $t$ Test). 


\section{Cre(-) control, 8M}
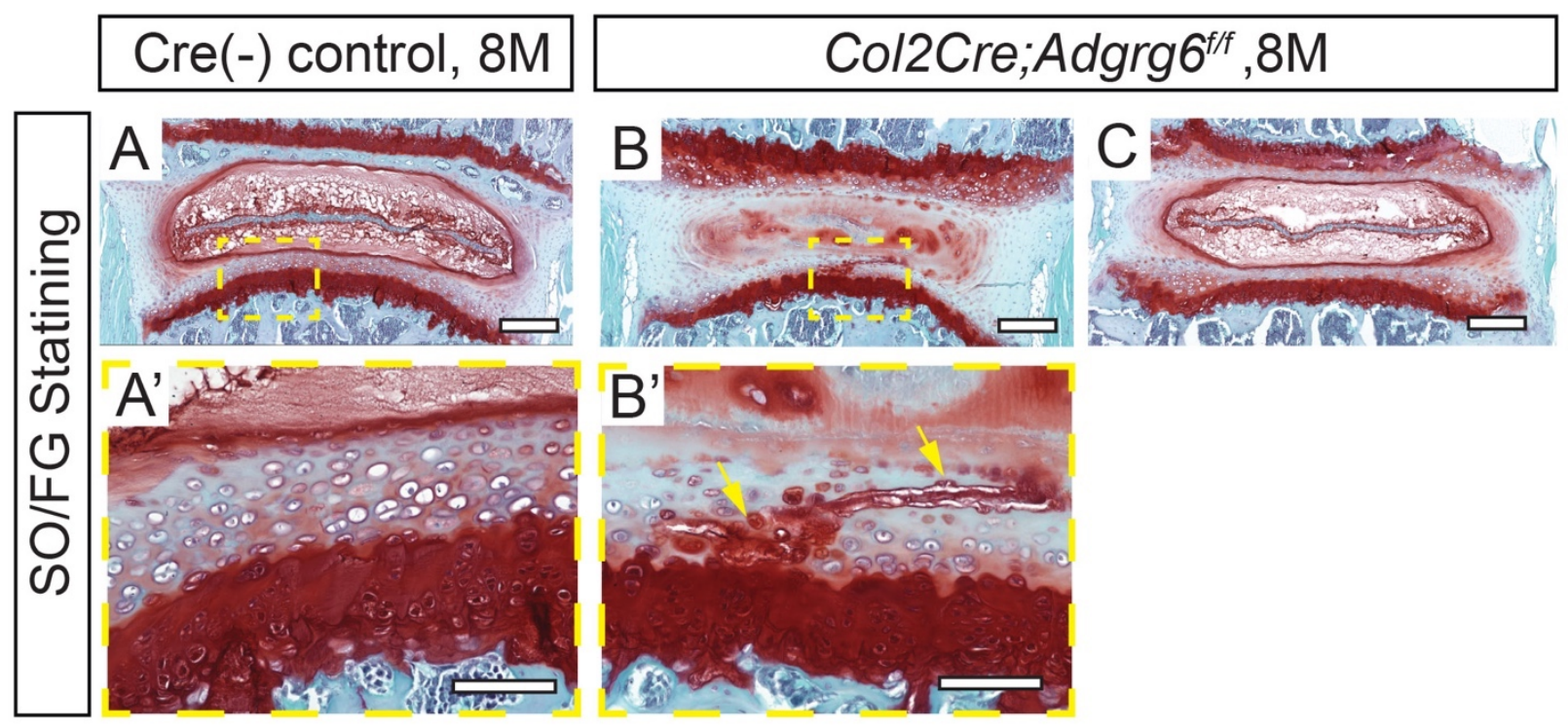

Supplemental Figure 7: Col2Cre;Adgrg $6^{f / f}$ mutant mice display endplate-herniation of the IVD. (A-C) Representative medial-sectioned mouse IVDs stained with Safranin-O/Fast green (SO/FG) of Cre (-) control (A and $\left.\mathrm{A}^{\prime}\right)$ and Col2Cre;Adgrg6fff mutant (B, B', and C) mice by the age of 8 months. Endplate-oriented herniations is indicated with yellow arrows. These herniations are very hard to be captured by traditional two-dimensional histological analysis (B, which is out of the typical plane of section). $\mathrm{C}$ is an earlier section of the same mutant IVD as shown in B, looking completely normal. ( $n=3$ for each group.) Scale bars: $200 \mu \mathrm{m}$ in (A-C), and $50 \mu \mathrm{m}$ in (A', B'). 

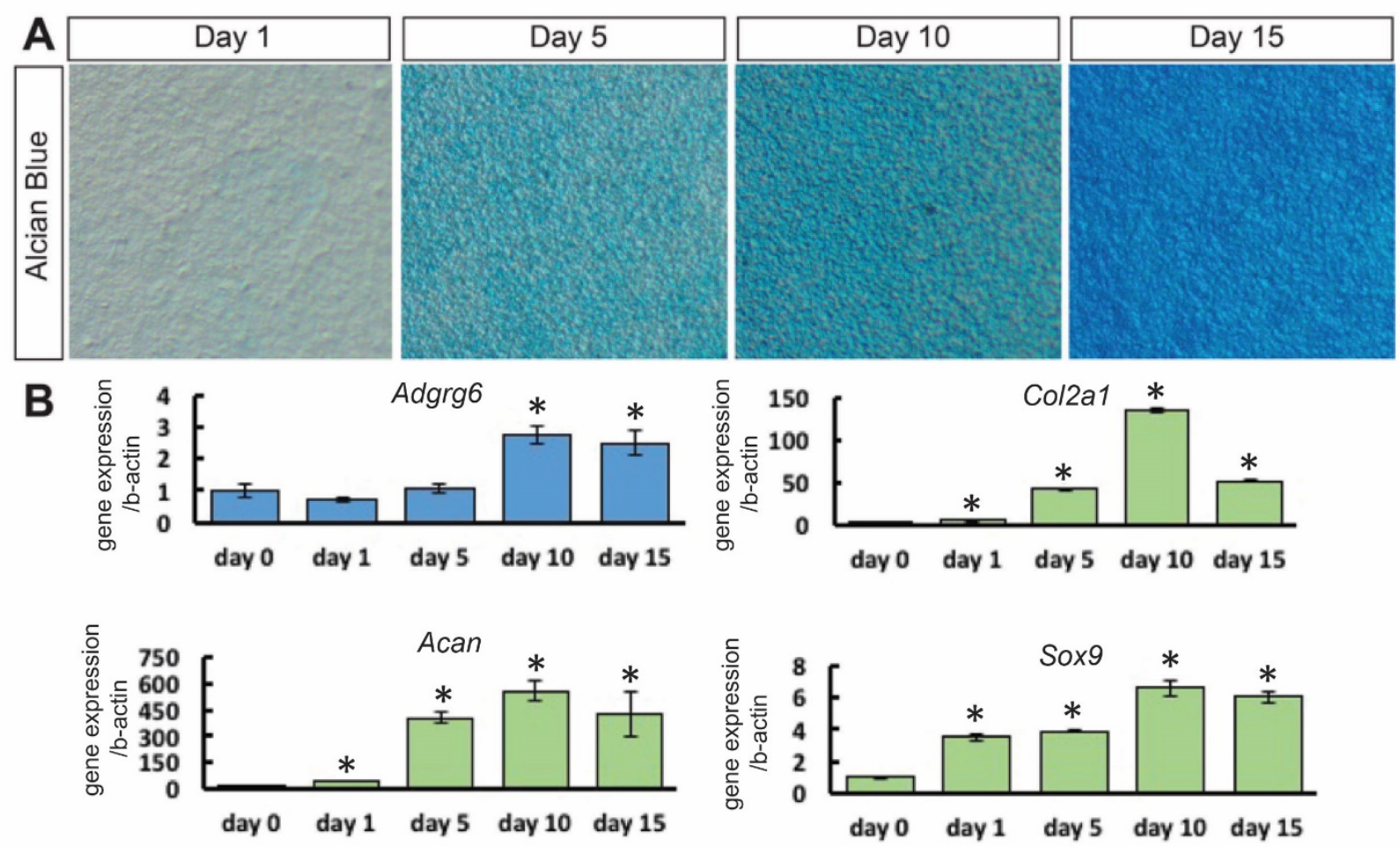

Supplemental Figure 8: Adgrg6 regulates ATDC5 cell maturation. (A) Alcian blue staining on ATDC5 cell culture during the maturation process. (B) Expression profiles of Adgrg6, Col2al, Acan, and Sox9 during ATDC5 cell maturation. The expression level of Adgrg6 was gradually increased alone with other chondrogenesis markers including Col2a1, Acan, and Sox9. $(n=3$ biological replicates and representative result is shown. Bars represent mean $\pm \mathrm{SD}$. $*_{p} \leq 0.05$, Student's t Test). 

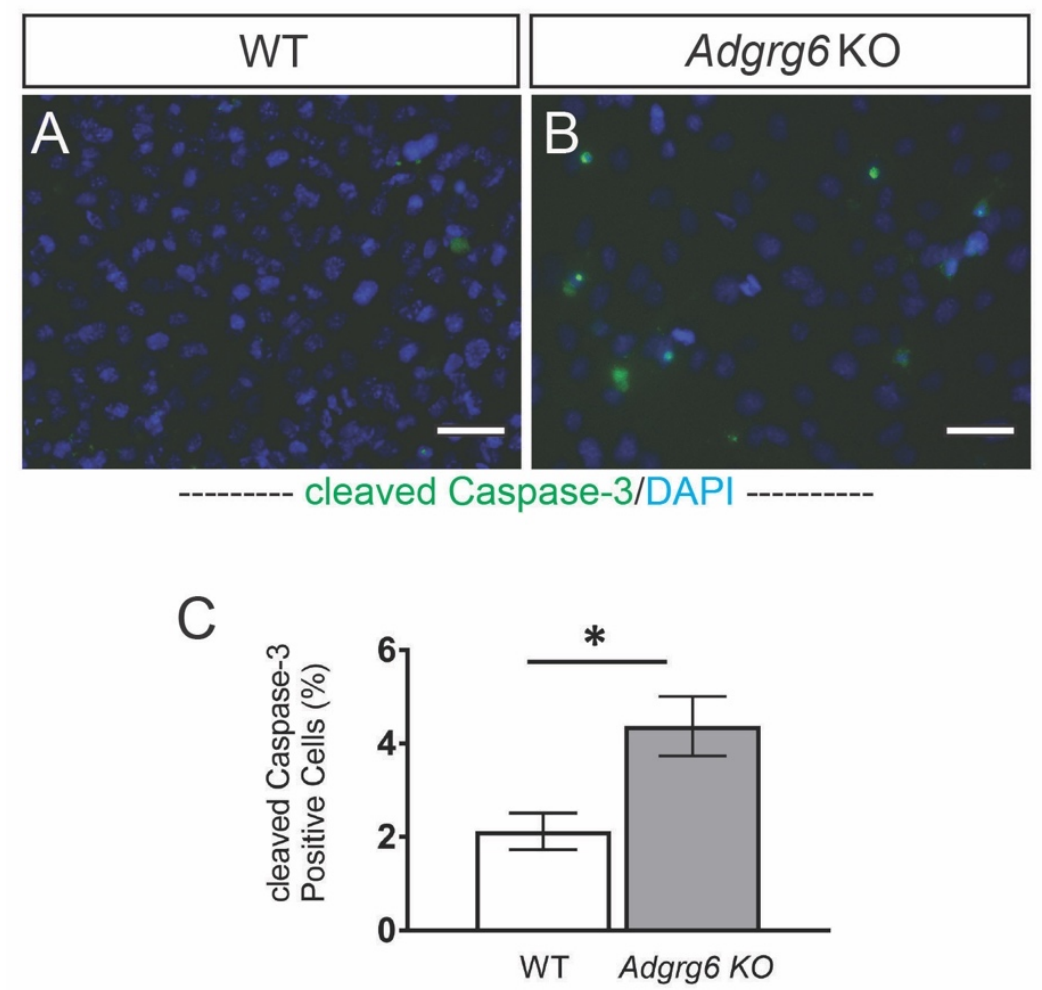

Supplemental Figure 9: Adgrg6 KO cells showed increased expression of apoptosis marker during maturation. (A, B) Immunofluorescence against cleaved-Caspace-3 (green) and DAPI staining (blue) and (C) quantification showing increased apoptosis in Adgrg6 KO cells during maturation (10 days). ( $n=3$ biological replicates and representative result is shown. Bars represent mean $\pm \mathrm{SD} .{ }^{*} \mathrm{p} \leq 0.05$, Student's t Test). Scale bars: $50 \mu \mathrm{m}$ in $(\mathrm{F}, \mathrm{G})$. 


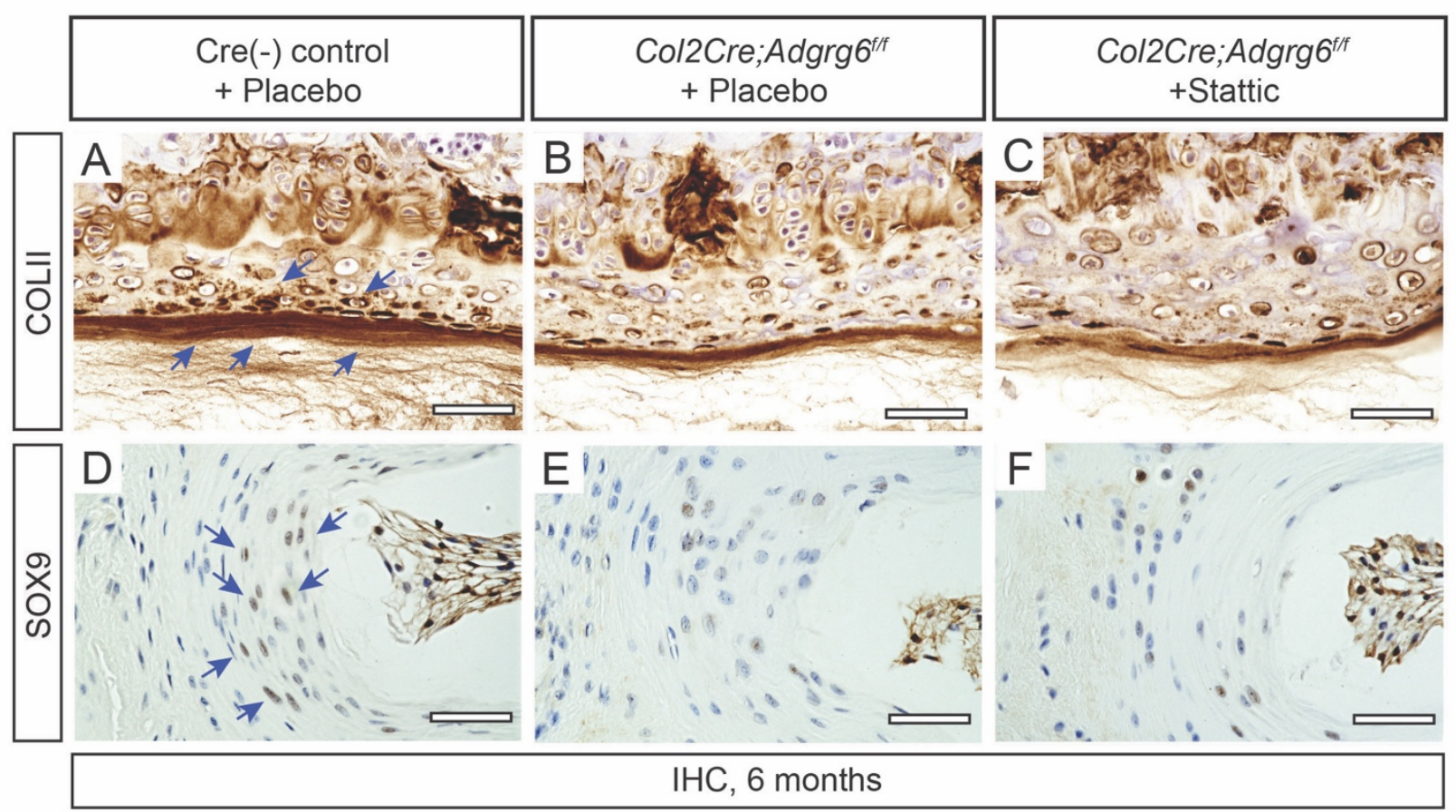

\section{Supplemental Figure 10: Inhibition of STAT3 activation by Stattic does not affect the} expression of COLII and SOX9 in Col2Cre;Adgrg6 ${ }^{\text {f/f }}$ mutant mice. (A-F) IHC analysis of COLII and SOX9 in 6-month-old Cre (-) control (A, D) and Col2Cre;Adgrg6fff mutant mice with $(\mathrm{C}, \mathrm{F})$ or without $(\mathrm{B}, \mathrm{E})$ Stattic treatment. Reduced COLII and SOX9 expression was observed in Col2Cre;Adgrg6fff conditional mutant mice compared with Cre (-) control (A, D, blue arrows), but no significant improvement was observed after Stattic treatment $(\mathrm{C}, \mathrm{F})$. ( $n=3$ for each group). Scale bars: $50 \mu \mathrm{m}$ in (A-F). 


\section{Supplemental Tables}

Table S1. Differential gene expression RNA-sequencing analysis from P20 IVD derived from Col2Cre;Adgrg6 $6^{f / f}$ and Cre(-) wild-type littermates.

Table S2. GO term analysis of P20 IVD derived from Col2Cre;Adgrg $6^{f / f}$ and Cre(-) wildtype littermates.

Table S3. qPCR primers used in this study.

\begin{tabular}{|l|l|l|}
\hline $\begin{array}{l}\text { Gene ID } \\
\text { (Mouse) }\end{array}$ & Forward primer (5'-3') & Reverse primer (5'-3') \\
\hline B-actin & AGATGTGGATCAGCAAGCAG & GCGCAAGTTAGGTTTTGTCA \\
\hline Adgrg6 & CCAAAGTTGGCAATGAAGGT & GCTGGATCAGGTAGGAACCA \\
\hline Sox9 & AGGAAGCTGGCAGACCAGTA & CGTTCTTCACCGACTTCCTC \\
\hline Col2a1 & ACTGGTAAGTGGGGCAAGAC & CCACACCAAATTCCTGTTCA \\
\hline Acan & CGTGTTTCCAAGGAAAAGGA & TGTGCTGATCAAAGTCCAG \\
\hline Prg4 & AAACAGCCAATAAGAGCCCTTGGC & TGGCTTTGACTTGGCTTTGACACG \\
\hline Col10a1 & CTTTGTGTGCCTTTCAATCG & GTGAGGTACAGCCTACCAGTTTT \\
\hline Col1a1 & GCATGGCCAAGAAGACATCC & CCTCGGGTTTCCACGTCTC \\
\hline Col3a1 & CTACACCTGCTCCTGTGCTT & CCAGTTGGACATGATTCACA \\
\hline Mmp13 & AGACTGGTAATGGCATCAAGG & GCCATTTCATGCTTCCTGATG \\
\hline Adamts4 & CAAGCAGTCGGGCTCCTT & GATCGTGACCACATCGCTGTA \\
\hline Adamts5 & CCAGTTGTACAAAGATTATCGGAACCT & GTTGCTCCTTCAGGGATCCT \\
\hline I11a & GCACCTTACACCTACCAGAGT & AAACTTCTGCCTGACGAGCTT \\
\hline II1b & GCAACTGTTCCTGAACTCAACT & ATCTTTTGGGGTCCGTCAACT \\
\hline II6 & CGGCCTTCCCTACTTCACAAGTCCG & CAGGTCTGTTGGGAGTGGTATCC \\
\hline Tnf & GCACCTTACACCTACCAGAGT & AAACTTCTGCCTGACGAGCTT \\
\hline Socs3 & ATGGTCACCCACAGCAAGTTT & TCCAGTAGAATCCGCTCTCCT \\
\hline
\end{tabular}

